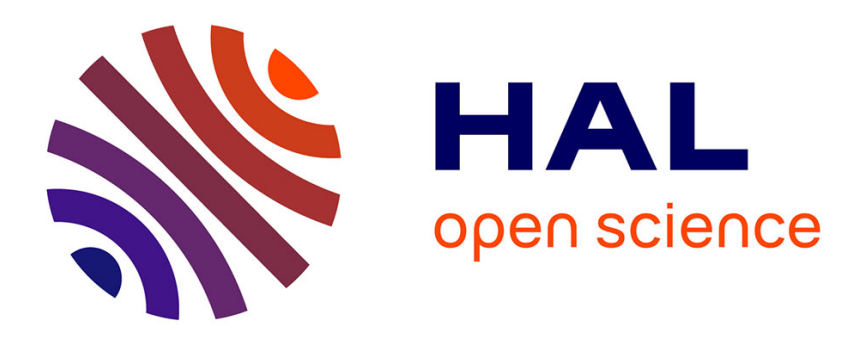

\title{
Le contrôle de la mobilité des personnes dans l'empire romain
}

Claudia Moatti,

\section{To cite this version:}

Claudia Moatti,. Le contrôle de la mobilité des personnes dans l'empire romain. Mélanges de l'Ecole française de Rome - Antiquité, 2000, 112 (2), pp.925-958. halshs-00660677

\section{HAL Id: halshs-00660677 https://shs.hal.science/halshs-00660677}

Submitted on 17 Jan 2012

HAL is a multi-disciplinary open access archive for the deposit and dissemination of scientific research documents, whether they are published or not. The documents may come from teaching and research institutions in France or abroad, or from public or private research centers.
L'archive ouverte pluridisciplinaire HAL, est destinée au dépôt et à la diffusion de documents scientifiques de niveau recherche, publiés ou non, émanant des établissements d'enseignement et de recherche français ou étrangers, des laboratoires publics ou privés. 
"Interdire le séjour d'une ville à des étrangers est proprement inhumain" Cicéron, de officiis, III, 11, 47

'Il est un désordre de police d'autant plus odieux qu'il tient à tous les arts de la tyrannie et prive l'homme de premier, du plus juste de ses droits, celui de respirer l'air qui lui plaît sans demander la permission d'un maître qui peut la lui refuser : celui des passeports" Discours de Peuchet sous la Révolution française ${ }^{1}$

Dans l'antiquité l'éloge de la patrie est un lieu commun. Si les traités sur l'exil mettent l'accent sur la liberté que constitue le départ² ${ }^{2}$ si les Stö̈ciens ont, par cosmopolitisme, exalté la patrie commune, le mundus, que les hommes partagent avec les dieux, plus nombreux sont ceux qui disent leur attachement à leur cité : “Ah, Rome, Rome, c'est là qu'il faut vivre !", écrivait Cicéron à son ami Marcus Caelius Rufus. À quoi fait écho Ovide : "Je songe à Rome, à ma maison, je regrette les lieux et tout ce qui reste de moi dans la Ville à jamais perdue" ou encore Lucien : "Personne... n’est séduit par les merveilles abondantes qu'offrent les autres pays au point d'oublier sa patrie"’. À la différence des Juifs pour qui partir est l'acte fondateur par excellence ("Va-t-en de ta terre et de ton parentage et de l'habitation de ton père vers la terre que je te ferai voir"'4), quitter sa patrie est un grand malheur ou au moins un ennui aux yeux des Grecs et des Romains. Ainsi Platon, qui considère le mélange des peuples comme inévitable mais aussi comme une des causes de la dégradation des mœurs ${ }^{5}$, propose dans Les lois une série de mesures visant à réglementer l'accueil des étrangers ${ }^{6}$ et surtout à limiter la mobilité des citoyens ${ }^{7}$ : "Personne au-dessous de quarante ans d'âge n'aura le droit de quitter le pays sous aucun prétexte et pour aucune destination que ce soit ; personne en outre n'y sera jamais autorisé en tant qu'homme privé, mais seulement pour servir son pays en qualité de héraut, d'ambassadeur ou d'observateur". Après lui, Plutarque reconnait qu"'il n'est ni bien ni

\footnotetext{
${ }^{1}$ Cité par G.Noiriel, 1998

${ }^{2}$ Voir par exemple l'analyse de Crifo, 1971, ch.1.

${ }^{3}$ Dans l'ordre, Cic., Fam., II, 12 (lettre CCLXV, juin 50). Le texte se poursuit ainsi : "Il n'y a point de séjour à l'étranger — j'en ai ainsi jugé dès mon adolescence — qui ne soit obscur et misérable quand on peut à Rome faire briller son activité"; Ovide, Tristes 3, 2 ; Lucien, Eloge de la patrie, 5 ; cf aussi Philon, de spec.leg., I, 68.

${ }^{4}$ Genèse.12,1-3 analysé par Philon d'Alexandrie, de fuga, 1 sq.

${ }^{5}$ Une idée reprise par Cicéron, de rep., II, 4, 7: "Rien n’a plus contribué à ... renverser Carthage et Corinthe que ces voyages sans fin qui dispersaient les citoyens (error et dissipatio civium) : en effet, poussés par l'amour du commerce et de la navigation, ils avaient délaissé l'agriculture et l'entraînement militaire".

${ }^{6}$ Lois XII, $952 \mathrm{~d}$.

${ }^{7}$ Lois XII, $950 \mathrm{~d}$.
} 
honnête d'abandonner sa cité pour aller en habiter une autre"8. De fait l'homme est attaché à son lieu d'origine par des liens historiques, familiaux, par les cultes domestiques, par les munera qu'il doit accomplir.

Dans un tel contexte, étudier les déplacements de population peut paraitre marginal. Et pourtant la mobilité dans l'empire romain est une donnée incontestable. Conférenciers, marchands, hommes d'affaires, mais aussi officiels et pèlerins ${ }^{9}$, exilés, fugitifs et mendiants ${ }^{10}$, étudiants et aventuriers quittent leur contrée, parcourent les provinces pour un temps ou pour de longues durées ${ }^{11}$ : outre la documentation épigraphique (inscriptions funéraires, dédicaces ou encore ostraka), les sources littéraires attestent largement ces migrations internes et le caractère cosmopolite des grandes villes ${ }^{12}$. À Smyrne, "on trouve des Ioniens, des Lydiens, Cariens [... ] Mais la ville attire aussi des gens de Cappadoce, des Assyriens, des Egyptiens, Phéniciens...et toute la jeunesse d'Athènes." 13 Tel est aussi le cas de Rome et sans doute de toutes les grandes villes d'époque romaine ${ }^{14}$. Une synthèse sur la question serait bien venue, mais le problème qui nous intéressera est un peu différent.

\section{La notion de contrôle}

Sans chercher à établir de manière plus précise la réalité ou la finalité des mouvements de population, les logiques migratoires et leur diversité, on voudrait se demander si les personnes circulaient librement à l'intérieur de l'espace romain ou si, au contraire, tant au niveau impérial que municipal, cette mobilité était contrôlée, et, selon les époques, sous quelles formes : les individus disposaient-ils de documents pour prouver leur identité (sociale ou autre) et les autorités pouvaient-elles vérifier leur validité, ou bien

\footnotetext{
8 Plut. Sur l'exil, 8.

${ }^{9}$ Chrétiens, mais aussi païens et Juifs : parmi les privilèges accordés par César aux Juifs, il y a la liberté de pèlerinage et la protection des caravanes qui chaque année apportaient l'argent sacré au Temple. Tout cela implique une série de contrôles et donc de directives adressées par César puis par les empereurs aux gouverneurs des provinces, à charge pour eux d'en aviser par écrit les villes concernées par ces déplacements (cf par exemple Jos. Ant.Jud., XVI, 6, 2-4 (162-7) ; 6, 6-7(171-72) ; autres sources dans J.Juster, 1914, I, p.357 sq). Sur les pèlerinages chrétiens, la bibliographie est importante mais, à ma connaissance, n'aborde jamais l'aspect documentaire : cf L.Casson, 1974 ; E.D.Hunt, 1984. En revanche, les modernistes se sont penchés sur la question : cf en dernier lieu Ph.Boutry et D.Julia éd; 2000, notamment l'article de D.Julia, p.26-37.

$10 \mathrm{Il}$ faudrait étudier la façon dont on transmet l'information concernant les esclaves fugitifs, les brigands... Dans ce sens, cf P.Oxy XII 1408 (édit de 210-214) réprimant le brigandage : il est demandé d'afficher l'édit “dans toutes les capitales et tous les lieux importants des nomes". Cf aussi D.11.4.1.8a (Ulp., lib.I ad ed.) : “on doit donner aux magistrats les noms (nomina) et les signes distinctifs (notae) des fugitifs et de ceux auquels ils appartiennent afin qu'on les identifie facilement".

11 Cf J.Hatzfeld., 1919 ; A.J.N.Wilson, 1966.

12 La bibiographie est considérable. Citons pour mémoire L.Robert, 1946 ; les mises au point de la revue Ktèma,13-14, 1988-89 ; C.Ricci, 1994 ; cf aussi S.E. Sidebotham, 1986, p.83 sq.

13 Philostrate, Vit.Soph., I, 21, Scopelianos de Clazomène ; cf aussi II, 26 Héraclide de Lycie.

${ }^{14}$ Sur Rome, Cic., Comment. petit., 54 ; App. B.C., II, 120 ; D.I.2.2.28 où la création du préteur pérégrin à Rome est référée à une multa turba peregrinorum : autres sources dans C.Moatti, 1997, p.59 sq.. Les sources épigraphiques relatives aux associations mais aussi la présence des cultes étrangers constituent évidemment des indices certains : cf G.La Piana 1927 ; L.Ross Taylor, s.d. ; F.Coarelli, 1982. Pour d'autres exemples que Rome, cf C.Nicolet éd., 2000, particulièrement l'article de.F.Burkhalter et A.Martin, p.253 sq sur Alexandrie.
} 
avaient-elles d'autres moyens de surveiller ces flux migratoires? La notion de liberté de circulation fut-elle même conceptualisée ${ }^{15}$ ?

De telles questions n’ont pour ainsi dire jamais été abordées, du moins sous cet aspect "documentaire". Pourtant, si l'enquête présente des difficultés considérables, elle touche à des aspects à la fois sociaux, politiques et militaires. En effet, le contrôle des personnes met en jeu la préservation de l'ordre public à l'intérieur d'un espace donné16, la définition de la territorialité et de la souveraineté dans la mesure où la surveillance des

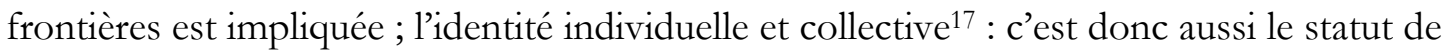
l'autre, de l'étranger qui y est impliqué.

Evitons tout anachronisme cependant. Les procédures de contrôle évoluent selon les sociétés, elles en sont même indéniablement le reflet. Il ne s'agit donc pas de présupposer un contrôle systématique des personnes dans l'empire ni de rechercher les ancêtres de nos passeports individualisés, mais de tenter de définir, si elles ont existé, les formes romaines de ce contrôle et leur évolution. Sans doute les sources suggèrent-elles à première vue l'absence de tout règlement général ainsi que nous incite à le croire L'éloge de l'empereur du Pseudo-Aristide, daté du règne de Philippe l'Arabe : "La possibilité de se rendre sans crainte là où l'on veut n'est-elle pas totale pour tous ?...Quels passages sont-ils interdits aux franchissements ? Sur la mer y-a-t-il des détroits fermés ?’(\$ 34). On pourrait donc cesser là toute enquête et décider, comme l'ont fait tant de chercheurs, que la nature même de l'administration romaine imposait une liberté de circulation ${ }^{18}$. Le raisonnement de M.R.Marrus à propos de l'immigration en France en 1870 s'impose aussi pour l'Antiquité : "Même en admettant que les autorités désirent chasser les étrangers elles n'ont que peu de chance d'y parvenir. Sans empreintes digitales, photographie d'identité, fichier efficace, ni réseau policier modernes, les réfugiés peuvent assez facilement franchir les frontières et vivre à peu près en paix dans le pays qu'ils ont choisi”'19. Mais, outre que Marrus n'envisage qu'un aspect du problème, il me semble nécessaire de poser autrement les questions.

\section{Quelques postulats}

D’abord on renoncera à toute approche trop générale : aucune source, nous l'avons dit, ne suggère l'existence d'un contrôle global de la mobilité. On peut même partir du principe que la liberté de circuler est le propre du citoyen et de l'homme libre. En revanche, on cherchera à repérer les contrôles particuliers et leurs modalités.

\footnotetext{
15 Comme se le demandait Nordmann pour la Renaissance (1987). Il faudrait en faire l'enquête systématique en partant de ce grand texte du de officiis de Cicéron cité ci-dessus et de toute la littérature sur l'exil analysée par G.Crifo, 1971.

16 Sur cette problématique, cf D.Nordman, 1987, qui l'applique à l'Ancien Régime.

17 Tel est notamment le sens du passeport établi sous la IIIe République : dans le contexte du développement de l'Etat-Nation en tant que communauté de membres, il devait "matérialiser l'appartenance des individus à cette communauté (la nation) en garantissant leur identité personnelle” (G.Noiriel, 1998, p.77-78).

18 W.Williams,1967 ; J.Gagé, 1971 ; M.Reinhold, 1971, p. 276 ; S.Mitchell, 1976, p. 196.

${ }^{19} 1986$, cité par G.Noiriel, 1988, p.75. Les remarques de Noiriel sur l'indigence des contrôle en France au XIXe s sont très pertinentes pour nous.
} 
Ensuite on posera qu'un document qui atteste l'identité n'est pas nécessairement d'origine publique : les lettres privées de recommandation qui concernent soit des clients qui se déplacent ${ }^{20}$ soit des serviteurs ${ }^{21}$, soit des hôtes, les tessères d’identité ou d'hospitalité22, équivalent des symbola pour les cités grecques ${ }^{23}$, les contrats d'affaires sont autant de moyens pour un individu d'être reconnu, et ce n'est pas rien, vu l'importance des réseaux de sociabilité dans l'empire. De plus, il faut garder à l'esprit que les insignes distinctifs extérieurs (vêtements, anneau d’or...), et surtout la déclaration orale (la professio) jouent également un grand rôle ${ }^{24}$.

Si la problématique ainsi définie est assez claire, l'enquête présente de nombreuses difficultés, tant la matière est éparse et le dépouillement lent. Impossible en effet de constituer un corpus de sources bien déterminé, car l’information concerne tous les domaines. Impossible donc de présenter une approche exhaustive de la question : il faudrait tout relire, et sur une très longue période, depuis la République jusqu'au VIe siècle au moins, dans la mesure où les textes juridiques tardo-antiques attestent nettement un renforcement des contrôles. En l'absence d'archives directes, toutes les sources doivent être convoquées : les correspondances, la littérature de voyage et de pèlerinage, mais aussi les textes de droit, les traités ${ }^{25}$, les inscriptions concernant les légations, ou encore les tarifs commerciaux. La surveillance de la circulation des biens est en effet abondamment étudiée et peut nous être de quelque utilité, ne serait-ce que pour mieux connaître le contrôle des

\footnotetext{
20 Attestées par la correspondance de Cicéron, les lettres de recommandation existent encore au IVe.s. Cf Symmaque à Promotus (Ep., 76) : “comme notre client Parégorius préparait son retour en Afrique et me réclamait vivement une lettre de cette sorte, $\mathrm{j}$ 'ai veillé à satisfaire son désir, sachant qu'auprès de qui m'aime ni sa requête ne manquerait d'être bien reçue, ni mes propos ne seraient inefficaces."

${ }^{21}$ Cf Symmaque à Ricomer (Ep., 67) en 388-91 : "Firmus qui fut attaché à ma maison (domesticum meum) s'est honorablement acquitté de son temps de service. Il me convenait donc de l'aider de mon témoignage, pour qu'en lui facilitant votre approche, il parvînt à se faire connaitre de vous".

22 Telle la fameuse plaquette d'ivoire portant le nom d'un marchand étrusque de Sulcis ou celle appartenant à un homme qui se dit de Carthage (E.Benveniste, 1933). La bibliographie sur le sujet est dans M.Gras (1985, p.206 sq) que je remercie vivement pour avoir attiré mon attention sur cette documentation, ainsi que sur l'ouvrage de F.Cordano (1992) concernant les tessères publiques de Camerina. Il faudrait en effet revoir, dans cette perspective, le dossier de ces "tessères d'identité" servant comme attestations de présence aux assemblées ou d'identité en général et portant le nom, le patronyme et la phratrie des citoyens. Dans quelle mesure toutefois ces documents à fonctionnement interne étaient-ils de quelque utilité dans un autre contexte géographique?

23 Cf Ph. Gauthier, 1972.

24 Cela explique aussi la facilité avec laquelle on usurpait un statut comme en témoigne l'histoire, rapportée par Suétone (Rhet.3) de ces marchands d'esclaves qui bernèrent les douaniers en faisant endosser à un jeune esclave la bulle et la prétexte.

${ }^{25}$ Que nous apprennent les traités, par exemple, sur la question du droit de passage sur un territoire romain de troupes étrangères, de la restitution des fugitifs ou simplement du droit de commercer? La question mériterait d'être systématiquement étudiée de même que serait utile une collection des textes de traités et plus généralement d'accords diplomatiques, parvenus jusqu'à nous sous forme littéraire sous forme épigraphique, comme cela a été entrepris pour la Grèce (cf S.Cataldi, 1983). Sur les droits de passages, voir le traité entre Rome et Méthymna (154 a.C.), 1.2-5 : "les Romains ne laisseront pas aux ennemis ou opposants du peuple de Méthymne le passage sur leur territoire ou sur celui qu'ils dominent” (Magie, II, 967-8 = Sherk, RGE, n³3, p.32) ; même chose dans le traité entre Rome et Astpalaia (105 av.J.C.) (RDGE 16B et trad. dans Sherk, RGE n`53 p.57, 11.30-32 ; 35-37) ; ou dans celui passé avec Mitylène (RDGE 26 et RGE 97, col c, 14) ; cf aussi. Liv.38,38, 2. ; M.R.Cimma, 1976, p. 253 sq sur les traités avec les Parthes. Sur les formes de traités à l'époque républicaine et impériale cf J.L.Ferrary, 1990 et F.Grelle, 1990.
} 
marchands ${ }^{26}$. Mais surtout pour nous demander si les Romains distinguaient la liberté de déplacement et la licence pour les marchandises, si le contrôle des personnes a existé indépendamment du contrôle des biens, s'il a eu une autre dimension qu'économique ou fiscale 27 : par exemple, il semble que le contrôle militaire aux frontières était très étroitement lié au fonctionnement des douanes.

Enfin, mais la liste n'est pas exhaustive, les sources chrétiennes nous sont précieuses dans la mesure où les persécutions ont été accompagnées de diverses procédures de contrôle. Dans tous ces cas, cependant, il importe de différencier les provinces, les époques, en distinguant le contexte impérial du contexte municipal.

Une autre difficulté de l'enquête provient de l'absence quasi-totale de mots clés qui rendent compte de toutes les situations : rien d'équivalent dans les sources à notre vocabulaire moderne, au "passeport" ou à la "carte d'identité" — et cette absence a sans nul doute une signification. Différents champs lexicaux méritent cependant d'être dépouillés : celui de l'autorisation, de la permission, du congé, de la mission (comme apostolon, diploma, commeatus, par exemple) pour tenter de voir quels termes désignent un document écrit ou quel autre désigne une autorisation formelle simplement orale ; celui du voyage (transitus, exitus...) ou encore celui des beneficia ou privilèges (pour faire valoir une immunité fiscale devait-on produire des documents ?). Cela tout en sachant bien, comme le rappelle Sénèque ${ }^{28}$, que toutes les réalités ne possèdent pas nécessairement un mot spécifique pour les désigner.

\section{Les trois termes de l'enquête}

Ces difficultés établies, l'enquête, dont nous ne présenterons plus loin les premiers éléments, devra se dérouler sur trois plans. Tout d'abord le plan municipal. En temps ordinaire, en dehors des périodes de crise alimentaire, l'entrée dans Rome ou dans n’importe quelle ville de l'empire était-elle contrôlée et sous quelle forme ${ }^{29}$ ? Si le statut des résidents est assez bien connu (encore que tous les aspects “documentaires” de leur séjour ne le soient pas), qu'en était-il des étrangers de passage ? Des documents étaient-ils émis pour les autorisations de résidence ou les interdictions de séjour? Quel rôle jouaient dans

\footnotetext{
26 Voir dans cet esprit, l'excellent article d'A.Bresson, 1994 (je remercie L.Migeotte de me l'avoir signalé).

${ }^{27}$ Le problème se pose par exemple pour les Juifs. À partir de Vespasien, le contrôle de leur identité est lié au fiscus judaicus. Il importe de rappeler ici le terrible témoignage de Suétone (Dom.12) : "La taxe sur les Juifs fut exigée avec une rigueur toute particulière ; on y soumettait soit les prosélytes qui vivaient à la façon des Juifs sans l'avoir déclaré (improfessi), soit les personnes qui, en dissimulant leur origine (origine dissimulata), s'étaient dérobées aux tributs imposés à cette nation. Je me souviens d'avoir vu, quand j'étais adolescent, un agent du fisc (procurator) assisté d'un très nombreux conseil, examiner un vieillard de 90 ans pour voir s'il était circoncis". Le texte apprend donc que pour des raisons fiscales, les Juifs devaient se déclarer (par professio) aux autorités locales. Mais avant la taxe ? En Egypte ptolémaique et augustéenne, il semble qu'on ait dénombré les Juifs mais en était-il de même ailleurs, à Rome par exemple. Sur cette question, quelques éléments de réponse se trouvent dans S.Cohen, 1993, p. 1 sq. Corollairement, se pose le problème du contrôle des expulsions hors des villes (cf plus bas, notes 33-34).

${ }^{28}$ De ben., II, 34.

${ }^{29}$ Cf D.I.12.1.13 : "le préfet de la ville peut interdire le séjour dans la ville et dans les autres régions de son ressort". cf A.Chastagnol,1960, p.267 sq.
} 
le contrôle les stationes, qui à Rome représentaient les cités italiennes ou provinciales ${ }^{30}$ ? Les villes devaient, au moins pour des raisons fiscales, posséder les listes de pérégrins autorisés à séjourner, celle des corporati ou de certains groupes sociaux (la matrix des infames par exemple, bien connue à Carthage au IIe s. de notre ère ${ }^{31}$, mais on aimerait pouvoir préciser l'apparition de ces documents, leur nature surtout ainsi que leur diffusion dans les cités de l'empire — bref définir plus clairement la politique des villes dans l'accueil des étrangers.

Une telle étude permettra d'aborder le dossier des expulsions — philosophes, Juifs ou autres. Si une bibliographie non négligeable porte sur leurs causes, les aspects administratifs ne sont pas abordés. Or l'expulsion ne suppose-t-elle pas un contrôle pour être efficace, et surtout un moyen d'identifier les populations concernées - par exemple, les Cisalpins, au terme de la lex Papia en 65 av.J.C. ${ }^{32}$ ou ou encore, en 41 de notre ère, tous les Juifs ${ }^{33}$ ? La réponse n'est cependant pas évidente. Peut-être doit-on simplement supposer un contrôle lâche et peu rigoureux comme le laisse entendre le P.Giss.40, III-IV : "les Égyptiens qui sont à Alexandrie, notamment les paysans qui sont venus de toutes les parties de l'Egypte et peuvent aisément être retrouvés, doivent être expulsés de la cité par tous les moyens... Les Égyptiens de naissance peuvent être facilement reconnus par leur langue, s'ils ont pris l'habillement et l'apparence des autres ; bien plus, par leur mode de vie et leurs coutumes moins civilisées, ils se dénoncent comme des paysans égyptiens".

L'expulsion était-elle donc laissée à l'appréciation des autorités ? On comprendrait ainsi le problème des usurpations d'identité et de statut ${ }^{34}$.

Dans ce cadre municipal, un autre dossier pourra être ouvert : il s'agit du séjour des professeurs et des étudiants. Prenons l'exemple de Libanius : nommé à la chaire de Constantinople et ayant reçu un acte de nomination ${ }^{35}$, il ne pouvait pas quitter la cité sans autorisation, sans "une notification écrite (ta grammata)" "36. En était-il de même de tous les professeurs nommés aux chaires impériales ? Le cas des étudiants était aussi très réglementé, en tout cas à Rome à la fin du IVe siècle. Une constitution bien connue de Valentinien I récapitule toutes leurs obligations : "à peine arrivés à Rome, ils doivent voir le

\footnotetext{
30 Sur le rôle des stationes à Rome, il n'existe à ma connaissance que l'étude de L.Moretti, 1958.

31 Cf A.Chastagnol, 1960, p.338-39 ; p.266 sq : sur les listes des corporations, des notati; voir aussi Tac. Ann. II, 85 : les prostituées, qui faisaient partie des infames, déposaient une déclaration auprès des édiles (licentiam stupri apud aediles vulgaverunt); cf aussi Tertullien, de fuga, 13 sur la matrix (registre) des infames, détenue à Carthage par les beneficiarii et curiosi et où figurent également les Chrétiens.

32 Dio, XXXVII, 9, 5.: la loi expulsait “tous les étrangers résidents à Rome, exceptés ceux qui habitaient l'Italie".

33 Sur l'expulsion des Juifs, cf Suét. Claud., 25, 5 et les commentaires de M.Sordi, 1995. Une expulsion bien analysée est celle des peregrini qui fut décidée par le préfet Symmaque en 383 (Ambroise, de off., III, 44-52) : cf E.Faure, 1965, p.540.; voir aussi L Cracco Ruggini, 1980. Sur d'autres expulsions, cf J.P.V.D.Baldson, 1979, p. $98 \mathrm{sq}$; A.Chastagnol, 1960, p.268.

34 Sur cette question, cf. M.Reinhold, 1971 ; pour l'époque moderner, voir par exemple D.Julia, 2000, p.31.

35 Autob., 37 : “Un décret (dogmata) m'enjoignit de rester sur place car on craignait que si j'étais libre de partir ..." ; cf aussi $\int 74$.

36 Autob., 95 : "L'empereur me permit de revenir (chez moi) mais sans que ce fût définitif.. Ayant reçu la notification (ta grammata) j'appris la nouvelle de la mort de ma cousine..."
} 
magister census et lui remettre la permission écrite d'étudier à Rome, délivrée par le gouverneur provincal : le dossier doit comporter le nom de leur ville, un certificat de naissance, des références attestant leur scolarité (eiusmodi litteras perferant ut oppida hominum et natales et merita expressa teneantur). Les censuales préparent des rapports annuels sur le nombre et les progrès des étudiants et ils envoient chaque année ces rapports à l'empereur." Les censuales faisaient partie des bureaux du préfet de la ville qui par ailleurs avait le droit d'expulser les jeunes gens. Le séjour ne devait pas se prolonger au-delà de leur vingtième année ${ }^{37}$., sans doute pour ne pas "voir les jeunes curiales fuir leur cité natale et les munera qui les y attendent" 38 . On ne sait pas de quelle époque date l'ensemble de cette réglementation ${ }^{39}$, mais elle atteste une volonté de mieux contrôler la mobilité des jeunes (il faut l'autorisation de la cité d'origine) mais aussi l'entrée de la capitale et de surveiller tout séjour temporaire. Elle reflète aussi le renforcement de la contrainte policière qu'attestent les mesures prises à l'encontre des mendiants, des aventuriers, des errants ${ }^{40}$. À la même époque, ne l'oublions pas, Saint Ambroise, dans des accents qui rappellent ceux de Cicéron, s'en prend à la cruauté du préfet de la Ville qui expulse les peregrini en cas de crise de ravitaillement : la cité, écrit-il, a aussi besoin du travail des "étrangers", c'est-à-dire, ici, de ceux qui ne sont pas de Rome ${ }^{41}$.

Le deuxième volet de l'enquête concerne les entrées d'individus sur le territoire de l'empire. Comme le montrent les études sur la frontière, il existait un grand flux transfrontalier qui incluait contrebandiers, mais aussi otages, ambassadeurs, commerçants, esclaves, journaliers, espions ${ }^{42}$... Les sources témoignent de prescriptions différentes selon les peuples et selon les régions. On peut ici rapidement rapprocher le passage de Tacite sur les Hermundures "seuls entre les Germains à commercer non pas sur la rive mais à l'intérieur et dans la plus brillante colonie de la province de Rhétie [Augsbourg]. Partout, sans surveillant (sine custode), ils passent le fleuve..." ${ }^{43}$ et une remarque d'Ammien Marcellin qui décrit un certain personnage Stachao, maure, "qui se déplaçait librement dans notre pays" avant qu'il fût prouvé qu'il était espion ${ }^{44}$. Peut-on parler pour autant, comme le fait W.Whittaker, d'une "libre circulation des barbares aux frontières" ? Le territoire romain avait-il des frontières “qui invitaient à entrer" et qui servaient à contrôler les flux plus qu'à

37 CTh XIV,9.1 ; à Beyrouth la limite d'âge est repoussée à 25 ans (CJ.X.50.1). Cf H.I.Marrou, 1981, p.112 ; sur l'a censibus, voir W.G.Sinningen, 1957, p. 73-76, qui signale les changements survenus à l'époque des Ostrogoths où la permission d'étudier à Rome était communiquée non plus au magister census mais au prior senatus; et A.Chastagnol, 1960, p.76 sq.

38 J.M.Carrié, 1975, p.1029.

${ }^{39}$ Cf déjà Ulpien à propos des étudiants (D.47.10.5.5) : Romae utique domicilium non habeat; et D.50.5.9 (Paul.lib.I Responsorum) : ceux qui enseignent à Rome sont excusés des emplois de leur patrie comme s'ils enseignaient dans leur patrie même."

40 Cf S.Mazzarino, 1951, p.244, cité par J.M.Carrié, ibid.; voir aussi V.Neri, 1998.

41 Voir note 34 ; Cic., de off., III, 11, 47

42 Cf V.Chapot, 1907, p.199sq ; cf aussi P.Salway, 1965, p.24sq.

43 Tac., Germ., 41 ; cf aussi Hist., IV, 64 et 65, 3 où Civilis s'adresse aux gens de Cologne avec qui il pactise : "nous supprimons les taxes et les charges qui pèsent sur le commerce; que l'on puisse passer librement chez nous (sint transitus incustoditi) mais de jour et sans armes."

44 28.6.3 : cf C.R.Whittaker, 1989, p. 104. 
les interdire ${ }^{45}$ ? Il semble qu'il faille distinguer là encore selon les provinces et selon les périodes. Par exemple, selon Tacite encore, les marchands germains ne pouvaient commercer dans le territoire romain que sous escorte et à certaines places ${ }^{46}$. Dion Cassius précise que Marc Aurèle interdit aux Quades d'aller dans les marchés sur le territoire romain ${ }^{47}$; que Commode limita à une fois par mois la rencontre entre marchands romains et Germains, dans un seul lieu et sous la direction d'un centurion ${ }^{48}$. On sait aussi par Augustin qu'au IVe s, en Tripolitaine, dans le pays des Arzuges, "les barbares engagés pour la conduite des charrois et la garde des récoltes prêtent serment (de retourner chez eux ?) en présence du décurion préposé au limes ou devant le tribun en jurant par leurs démons. Les propriétaires ou leurs fermiers engagent en toute sécurité les gardiens des récoltes au vu d'une lettre du décurion et les voyageurs engagent de même ceux dont les services leur sont nécessaires" ${ }^{\prime 9}$. Un témoignage à rapprocher de celui des ostraka trouvés dans les camps militaires sur le limes et qui font état d'un dénombrement des barbares passés par le poste de garde. Le recueil de Bu Njem présenté par R.Marichal livre des renseignements extrêmements intéressants : le document 71 porte qu'“un jour d'octobre ou de novembre, sont entrés à Golas des Garamantes portant des lettres pour le praepositus et conduisant 4 ânes, et 2 Egyptiens et un esclave fugitif nommé Gtasazeiheme Opter"50 ; au numéro 101 on trouve cette notice : "scias domine benisse a meos refuga Abban barbarus" ("sache, seigneur, qu'est venu auprès de mes hommes un transfuge barbare Abban") ${ }^{51}$... Ainsi, le travail des journaliers, le commerce aux frontières étaient soumis à une surveillance certaine. De tels renseignements firent-ils cependant et à quelles époques l'objet de relevés réguliers sur tout le limes ? Ce qui semble clair, c'est qu'à la fin du IIIe siècle, les contraintes se firent plus rudes $^{52}$. Par exemple, en 298, le traité entre Rome et la Perse indique que le commerce entre les deux populations n'est permis qu'à Nisibe ${ }^{53}$. Par la suite, cette mesure fut étendue à d'autres provinces. À la fin du IVe s., on trouve des comites commerciorum ${ }^{54}$, chargés des opérations commerciales entre les étrangers et l'Etat. Ces comites donnaient des autorisations pour se rendre dans les villes où ce commerce était autorisé. C’est dans leurs bureaux que devaient se faire enregistrer les marchands étrangers et il était interdit de les

\footnotetext{
45 Sur cette problématique, voir aussi les remarques de J.Napoli, 1997, p.109 sq.

46 Tac., Hist.IV, 64-65 : sint transitus incustoditi; Dio, 71, 15.

$4772,11,3$.

$4873,2,4$.

49 Augustin, Epist., 46-7 (trad. R..Rebuffat, 1977, p.412 et commentaire p. 412-13) ; cf aussi R.G.Goodchild, 1950, p.31 ; R.Marichal, 1992, p. 111 qui avec R.Rebuffat rapproche ce texte d'un ostrakon de Bu Njem $\left(\mathrm{n}^{\circ} 71\right)$, cité plus bas (note 51$)$.

${ }^{50}$ Cf R.Marichal, 1992, p. 109 sq ; cf aussi N¹47.

51 Trad.R.Marichal, id., p.110 et commentaire : "nos Garamantes apportent donc peut-être une lettre d'un praepositus voisin de celui de Golas les accréditant ainsi auprès de lui.".

52 S.J.De Laet, 1949, p. 456 ; cf aussi R.Andreotti, 1969, surtout p.243 sq.

53 Sur ce traité, cf R.Andreotti, id.; cf aussi C.J.IV 63, 4 (Constitution d'Honorius et de Théodose, 408 ou 409).

${ }^{54}$ De Ruggiero, DE II, 507 ; R.Andreotti, id, p.246.
} 
héberger sans autorisation ${ }^{55}$. Le code de Justinien abonde en notices relatives à la protection et à la surveillance du commerce. Nous retrouvons là une des caractéristiques de la société du Bas-Empire, plus policière, plus contrôlée que jamais.

Reste un troisième axe de recherche : la circulation à l'intérieur de l'Empire. À première vue, la question est vite réglée : d'une part les professions mobiles sont très attestées $^{56}$, d'autre part les sources ne font pas état d'un contrôle systématique. Le récit de Dion Chrysostome sur son errance dans le bassin méditerranéen confirme cette impression : “Errant pendant mon exil, j’ai visité autant de pays que j’ai pu, tantôt allant chez les Grecs, tantôt chez les Barbares, vêtu comme un vagabond... À la fin, j’arrivai dans le Péloponnèse et restant à l'écart des cités, je passai mon temps dans la campagne...Comme je marchais le long du fleuve Alphée, sur la route qui mène d'Héraea à Pise,...je me retrouvai dans un bois et une région sauvage, sans rencontrer quiconque ni être capable de demander mon chemin." ${ }^{57}$ Les témoignages autobiographiques, la littérature de voyage, les vies des sophistes vont dans le même sens ${ }^{58}$, ainsi que les Métamorphoses d'Apulée, "image de tous les irréguliers qui au cours des deux premiers siècles traînent sur les routes de l'empire ou s'abritent en des cantons reculés" 59 . Donc ces foules anonymes, personne ne les contrôle ? Ces individus qui passent d'une province à l'autre ne sont jamais observés ? Si un voyageur entre dans une cité, un marchand par exemple, s'il décide de s’y installer, il devra déclarer son identité comme résident, démontrer donc son origine, présenter des documents. Mais s'il se déplace, il n'aura à rendre compte de rien ?

Les Romains ont pourtant accordé une grande importance à tous les problèmes de communication, et d'abord pour des raison d'ordre public. De nombreux postes de gardes et garnisons étaient disposés dans tout l'empire pour réprimer le banditisme ${ }^{60}$, assurer la tranquillité des citoyens et protéger les voyageurs sur certaines routes ${ }^{61}$. mais aussi pour veiller à la sécurité du ravitaillement : une inscription de Saepinum (datée entre 169 et 172) montre des préfets du prétoire prenant parti pour les pasteurs contre les autorités citadines et les stationarii qui, suspectant ces derniers de vols de bestiaux, les soumettent à de rudes

\footnotetext{
${ }^{55}$ CJ IV , 63, 6). On peut citer aussi les constitutions relatives au contrôle des frontières maritimes confiées à des curiosi litorum et des custodes, chargés de dépister l'infiltration étrangère ou la contrebande $(C T h 6,29,10$ de Théodose en 412 ; et 13.5 .5 (329) et 17 (396) de Valentinien II ; cf J.Gaudemet, 1958, p.215 et W.Goffart, 1980.

56 Cf F.Millar, 1977, 456-63.

57 Premier discours sur la royauté, 50 ; cf aussi Discours 13.

58 Philostr., Vit. Soph. II, 3 ; II, 5 : Alexandre de Séleucie : “il passa la majeure partie de son activité à Antioche, Rome, Tarse, et aussi en Egypte et alla jusqu'au pays des Gymnosophistes” ; II, 8 ; II, 9.

59 J.Gagé, 1960, p.147.

${ }^{60}$ Par exemple une inscription d'Intercisa en Pannonie marquait le site d'un poste de garde de l'armée, construit sous le règne de Commode en 184 pour surveiller les endroits sujets "au passage clandestin des bandits" (per loca opportuna ad clandestinos latrunculorum transitus).

${ }^{61}$ Egérie, récit de voyage, 7,4; 9, 3 : “à partir de là, nous avons renvoyé les soldats qui nous avaient procuré une escorte, au nom de l'autorité romaine, aussi longtemps que nous circulions dans des régions peu sûres. Mais comme c'était maintenant une voie publique à travers l'Egypte, celle qui va de Thébaïde à Péluse, qui traversait la ville d'Arabia, il n'était plus nécessaire dès lors de déranger les soldats." Le récit d'Egérie raconte le long voyage effectué en 383 par cette femme jusqu'en Terre Sainte.
} 
contrôles..... ${ }^{62}$ Mais la correspondance de Pline révèle que ces garnisons n'étaient pas systématiquement mises en place. Dans une lettre adressée à Trajan, le gouverneur de Bithynie demande à l'empereur de l'autoriser à envoyer à Juliopolis, “où passent tant de voyageurs étrangers", une garnison de stationarii sous la direction d'un centurion, identique à celle qui était installée à Byzance. Trajan refuse, prétextant que Juliopolis est trop petite pour un tel déploiement ${ }^{63}$. L'entrée dans la province par Juliopolis était donc libre.

En réalité, les contradictions que l'on peut relever entre les différentes sources semblent bien témoigner, indépendamment des paramètres chronologiques, du manque d'unité dans la politique romaine. Le cas des exilés en fournit un autre exemple. Dans une de ses lettres, Pline demande à Trajan ce qu'il doit faire d'un homme qui, malgré un décret d'expulsion, est demeuré dans la province ; en revanche, Philon se plait à donner les détails de l'arrestation du préfet d'Egypte Flaccus : condamné à l'exil et assigné à résidence à Andros, il est escorté par les gardes jusqu'à l'île et présenté à la population locale : "ils le montrèrent à toute l'assemblée en la prenant à témoin de l'arrivée du banni dans l'île" (In Flacc 157). Sans doute les décrets d'exil étaient-ils toujours affichés dans les lieux concernés par la condamnation. L'information circulait en grande partie ainsi ${ }^{64}$, accompagnée de pressions exercées depuis Rome comme le révèle le récit de son exil par Cicéron : en 58, alors que la loi lui interdisant de séjourner à moins de 500 milles des côtes de l'Italie vient d'être votée, son ami C.Vergilius, gouverneur de Sicile, lui refuse le séjour dans sa province, "craignant de ne pouvoir résister seul à la tempête politique". Cicéron, alors à Vibo, décide de rejoindre Brindes : “tous les municipes qui s’étendent entre Vibo et Brindes...réussirent, non sans les plus grandes appréhensions, en raison des nombreuses menaces qu'ils reçurent, à assurer la sécurité de mon voyage"65. Puis, avant de s'embarquer, Cicéron reste caché près de Brindes pendant treize jours chez son ami M.Laenius Flaccus, "bien que l'on

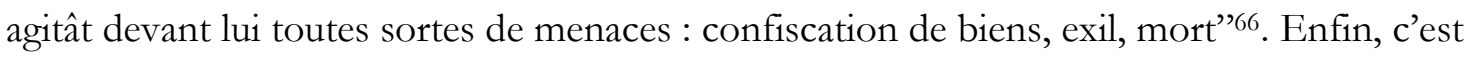
avec la complaisance de Cn.Plancius, alors questeur de Macédoine, qu'il peut illégalement résider dans cette province. C'est donc moins un contrôle direct que des pressions successives sur les autorités locales concernées (gouverneurs, cités, individus) qui, semble-til, permettaient de surveiller, sans grande efficacité d'ailleurs, un exilé politique ${ }^{67}$.

62 CIL IX, 2438. Mais cela a lieu dès l'époque d'Auguste : cf Suét. Aug. 32,1. On le sait aussi pour la route d'Antinoupolis à Berénice en Égypte (IGRRom I.1142) ; sur les burgarii, les postes de garde aux frontières chargés de la sécurité des voyageurs, cf l'inscription de 188 en Numidie : CIL VIII, 2495. Sur les beneficiarii, les stationarii, quelques mises au point intéressantes dans R.Mac Mullen, 1963, p. 57 sq. ; A.Giardina, 1977 ; B.Isaac, 1990, 1992 (2e éd), surtout p.78 sq ; p.103 sq ; W.Nippel, 1997 ; et pour le Bas-Empire, en dernier lieu, V.Neri, 1998, p.146 sq.

63 Cf Pline, Ep., 77-78 (et E.W.Black, 1995, p.11sq).

64 Plut., Cic., 32, 1 sq (876f-877a).

65 Cic., pro Planc., 40, 96.

66 Pro Planc., 41, 97.

${ }^{67}$ De même, c'est d'abord par des menaces, puis par des lois pénales qu'Auguste contraignaient les magistrats et les cités à respecter les privilèges reconnus aux Juifs (Jos.Ant.Jud.16,6, 2); cf J.Juster, 1914, I, p.238. 
Les trois axes de recherches que nous avons ainsi définis (l'entrée dans les villes, la circulation dans l'empire, le passage des frontières) présentent des difficultés réelles et il est probable que tous les problèmes soulevés ne trouveront pas de réponse : non seulement, nous l'avons dit, les sources sont lacunaires et parfois les indications trop partielles pour être interprétables, mais encore les informations restent dispersées : aussi, seule une enquête collective nous semble efficace à terme. Ces réserves étant posées, nous nous proposons de tenter une première expérience sur quatre cas particuliers : la circulation des officiels romains, le déplacement des ambassades, celui des marchands, enfin l'entrée et la sortie de l'Egypte. S’il n'a pas existé de réglementation générale sur la circulation des personnes, des mesures précises ont été prises à l'égard de certaines catégories de personnes ou de certains territoires.

\section{La circulation des officiels romains}

Selon Suétone, César voulait fixer la population en Italie et "assurer à la capitale épuisée une population suffisante" afin d'éviter la dispersion des fortunes et des gens (Iul., \42) : “il défendit à tout citoyen qui avait dépassé vingt ans ou n’en avait pas atteint soixante, à moins qu'il ne fût mobilisé, de rester plus de trois ans de suite loin de l'Italie ; aux fils de sénateurs de partir pour l'étranger, sinon dans l'état-major d'un général ou pour accompagner un magistrat..."

Des mesures de ce genre sont bien connues pendant toute l'époque républicaine. Outre les interdits religieux ${ }^{68}$ ou les restrictions liées aux périodes de guerre ${ }^{69}$, certaines fonctions impliquent un contrôle des déplacements : les tribuns de la plèbe dont la magistrature est liée à l'Urbs ne peuvent s'absenter de Rome que pour aller aux Monts Albains, l'un des trois jours des fêtes latines, et doivent rentrer avant minuit"70 ; les gouverneurs de province ne doivent pas quitter leur province sans autorisation sénatoriale ${ }^{71}$ et les sénateurs, dont la disponibilité est censée être totale, ne peuvent quitter l'Italie sans une legatio officielle ${ }^{72}$. Cette legatio libera était obtenue sur demande écrite faite au

\footnotetext{
${ }^{68}$ Liv. 28, 38,10 : interdiction faite au Pontifex Maximus de quitter l'Italie ; Liv. V, 53,13 interdiction pour le flamine de Jupiter de quitter Rome (cf.aussi Tac.Ann. III, 58,1 : Iunius Blaesus étant prorogé dans la province d'Afrique, Servius Maluginensis, flamine de Jupiter, demande à obtenir le proconsulat d'Asie, alléguant que c'était une idée fausse bien que répandue, que le flamine de Jupiter n'avait pas le droit de sortir de l'Italie, ajoutant que son statut n'était pas différent de celui des flamines de Mars et de Quirinus ; id, 71 et Val.Max,.I, 2.

69 Liv. XXXVI, 3,2 : en pleine préparation de la guerre contre Antiochus, "le consul P.Cornelius interdit à tout sénateur, à tous ceux qui avaient une voix délibérative dans le sénat et aux magistrats de second ordre, de s'éloigner de Rome à la distance d'une journée, et aux sénateurs de s'absenter de la ville à plus de cinq en même temps". Cf aussi Liv. 43.11.4. De même pendant la guerre civile en 49, il n'était pas permis de circuler librement (voir plus bas, note $\mathrm{OO}$ ).

70 Gell., N.A., III, 2 ; cf Liv. XXIX, 19.

71 Cic., II Verr., I.28, 73 ; D.I.18.15 (Marc.lib.I de indic.publ.) : “celui qui gouverne sa province ne doit point sortir si ce n'est pour remplir un vœu, de manière cependant qu'il ne puisse point coucher hors de sa province." Voir aussi Pis., 21.50 : la lex Cornelia de maiestate et la lex Iulia repetundarum de 59 renforçaient l'interdiction sauf s'il s'agissait d'une sortie rei publicae causa; cf A. Lintott, 1993, p.26 sq.

72 Cic., Phil., I, 2, 6. Sur la disponibilité du sénat, cf M.Bonnefond, 1989, p.199 sq
} 
Sénat, sans doute par l'intermédiaire des consuls ${ }^{73}:$ l'aspirant y indiquait les raisons de son voyage ainsi que sa durée ${ }^{74}$ - limitée à un an depuis le consulat de Cicéron ${ }^{75}$.

Avec l'augmentation du nombre des sénateurs provinciaux, la réglementation se fait plus sévère à l'époque impériale ${ }^{76}$. Auguste interdit à son tour aux sénateurs de quitter l'Italie " "sans permission ou mission", selon les mots de Dion Cassius qui place la mesure vers 29-2878. Exceptées de cette interdiction : la Sicile, puis sous Claude, en 49, la Narbonnaise ${ }^{79}$. Il en était de même au IIIe siècle d'après le même Dion : “aucun sénateur ne peut quitter le pays sans autorisation, sauf pour la Sicile et la Gaule Narbonnaise" ${ }^{80}$. À partir de Claude, c'est l'empereur qui délivre cette autorisation : comme le précise Suétone, Claude "se réserva le droit d'accorder des congés (commeatus) que l'on demandait jusqu'alors au sénat" "1. L'empereur sanctionna les délits : "il nota un grand nombre de personnes, certaines de façon inattendue et pour ce motif nouveau (causa novi generis) qu'elles avaient quitté l'Italie sans le prévenir ni lui demander congé (sine commeatu)" 82 . Une mesure qui fut modifiée à partir de Caracalla : la règle du double domicile étant instaurée, il fut permis aux sénateurs "de résider dans leur patrie provinciale, de se déplacer dans tout l'empire à leur volonté"83. Ajoutons toutefois que l'entrée en Egypte leur était toujours interdite sans autorisation spéciale ${ }^{84}$.

La legatio libera de l'époque républicaine et le commeatus impérial qui la remplace ${ }^{85}$ impliquent-ils la détention d'une lettre officielle ? Le mot commeatus est très vague. Outre ses sens concrets (action de transporter du ravitaillement, puis le ravitaillement lui-même mais aussi le voyage), il désigne le temps libre, donc le congé, c'est-à-dire la liberté de se déplacer pour un soldat ${ }^{86}$, un magistrat ou un administrateur ${ }^{87}$. Aucun des textes qui s'y rapportent ne permet toutefois de définir clairement s'il suggère l'existence d'un document écrit. Un passage de Tite Live pourrait le laisser entendre, mais l'expression employée est ambiguë. Il y est question d'Appius Claudius, l'un des decemvirs de 450 qui veut empêcher

\footnotetext{
73 Cic. Att.15.18,1 ; cf J.Suolahti, 1970, p.115 ; M.Bonnefond-Coudry, 1989, p. 370.

74 Fam., 11, 1, 2 (17 mars 44).

75 De leg., III, 18.

${ }^{76}$ Elle concerne aussi les décurions, qui “doivent demeurer dans les villes auxquelles ils appartiennent" (D.50.2.1.pr. Ulp., lib.2 Opin.).

77 Voir plus précisément : D.I.9.11 (Paul, lib.41 ad edict.) : "les sénateurs sont toujours censés demeurer à Rome, ce qui n'empêche pas qu'ils aient aussi un domicile dans leur patrie.”

78 Dion, LII, 42, 6 : ajvn mh; aujtov" tiv keleuvsúh hj; kai; ejpitrevyúh

79 Tac, Ann.XII, 23, 1.

80 Cf A.Chastagnol, 1977, p. 44.

81 Suét., Claud., 16 ; cf Dio, C.LX, 25, 6-7 (mesure de 45/46). Ce qui est confirmé par Pline, Ep.; X, 8, 6 et réponse de Trajan : Pline, Ep., X, 9.

82 Suét., Claud., 16.

83 D.L.1.22.6 (Paul., lib.I Sentent.) : senatores... liberum commeatum, id est ubi velint morandi arbitrium impetraverunt ; sur cette question, cf. A.Chastagnol, ibid.

84 Tac., Ann., II, 59, 3 ; Dio, LI, 17,1-2 ; cf M.A.Levi, 1924 ; A.Chastagnol, 1977, p. 44.

85 Cf Schol.Bobb. (ad Cic., Flacc., 86) : eas [legationes liberas] nunc commeatus appellamus. Mais le mot est déjà chez Tite Live au sens de congé, et surtout d'autorisation de se déplacer (I, 57,4 ; III, 46, 9 : cf plus bas).

86 Par ex.emple, D.49.16.1 ; cf TLL, s.v. commeatus.

${ }^{87}$ Cf Pline, Ep., X., 8, 6.
} 
Verginius, le père de Virginia, de venir à Rome : "Il écrivit de ne pas accorder de permission à Verginius" (ne Verginio commeatum dent) mais son ordre arriva trop tard ; Verginius avait déjà pris sa permission (commeato sumpto) et était déjà parti.”(III, 46,9). On pourrait imaginer que le terme sumere a un sens concret mais ce n'est pas toujours le cas ; d'autre part Festus définit le commeatus comme une durée : tempus quo ire, venire quis possit 88 . Il est donc difficile de savoir si le bénéficiaire du congé possédait une attestation nominale ${ }^{89}$. En revanche, l'autorisation impliquait un travail d'écriture dans les archives. Selon Végèce, "on enregistrait la date et la durée des congés des soldats (quando quis commeatum acceperit, vel quot dierum, adnotatur in brevibus)" ${ }^{\prime 00}$; il en était sans doute de même pour les autres officiels dont on peut supposer que la demande devait être conservée le temps du déplacement.

De ces congés, on doit distinguer nettement l'attribution des diplomata. Durant la guerre civile en 49, alors qu'il est interdit de circuler librement, Cicéron avoue à Atticus qu'il en a obtenu un ${ }^{91}$ : 'j'ai entendu dire que personne ne peut partir sans un diploma”, écrit-il à Atticus qui se demande comment il a eu une telle idée. “"Comme tu m’avais dit que tu pensais partir ${ }^{92}$, lui répond Cicéron, j’imaginais que tu t'en étais procuré un, ainsi que tu l'avais fait pour tes esclaves (pueris et diploma)"93.

Le mot diploma, qui désigne d'abord un document écrit ${ }^{94}$, quel qu'il soit, puis, plus précisément, à partir de Claude, le document plié en deux où se trouve l'attestation de citoyenneté et, éventuellement, d'immunité accordée aux vétérans" ${ }^{95}$, signifie aussi "saufconduit" 96 . Il peut s'agir d'un simple laisser-passer ou d'une reconnaissance du droit à circuler librement comme c'est le cas pour Cicéron en 49 ou pour les exilés autorisés à rentrer dans leur patrie ${ }^{97}$. Un diploma peut aussi contenir une clause donnant droit à des réquisitions dans les provinces — ce que sous l'Empire on nommera le cursus publicus-

\footnotetext{
${ }^{88}$ Festus, sv.Remeare, p. 344 L.

${ }^{89}$ En tout cas, celui qui bénéficiait d'une legatio libera n'avait pas de droit accès à l'hospitalité des provinciaux ni à un traitement privilégié. Lorsque son ami Anicius en reçoit une pour aller inspecter ses propriétés en Afrique, Cicéron écrit une lettre de recommandation au gouverneur d'Afrique Cornificus pour lui demander de veiller à ce qu'Anicius puisse bénéficier de tous les avantages officiels, y compris le droit de se faire précéder par des licteurs (Fam., 12, 21). Sur les rapports entre Anicius et Cicéron, cf E.Deniaux, 1993, p.447 sq.

90 Stratagèmes, II, 19.

91 Att., X, 17 (16 mai 49).

92 Dans une lettre précédente (Att.X, 13 du 7 mai 49), il est fait allusion au document qu'Atticus s'est procuré pour partir en Épire et donc traverser l'Adriatique : "toi aussi, je vois que tu es retenu par Curtius : je présume cependant que tu possèdes un sauf-conduit (ejkploun)", restitution vraisemblable, mais discutée dans CUF, t.6, p.105 note c. par J.Beaujeu selon qui le mot est adapté pour désigner la permission de quitter l'Italie en bateau (nous en reparlerons plus loin).

93 Att., X, 17.

94 Macr., Sat., I, 23, 14 : “ils consultent ce dieu de loin, en lui envoyant des billets munis d'un sceau (missis diplomatibus consignatis)."

95 Suét., Ner., 12. ; sur les diplômes militaires, cf notamment J.C.Mann et R.R.Roxan, 1988.

96 On trouve en grec divplwma ou divplh : PSI V, 446, 1l. 3 et 12.

97 Cic., Fam., VI,12,3 : “le diploma n'a pas été délivré sur-le-champ à cause de l'étonnante malhonnêteté de certaines gens [...], écrit Cicéron à Ampius Balbus mais le délai sera très bref [...]. En tout cas, Pansa m’en a donné l'assurance et s'est même fait fort de l'obtenir très rapidement”. Même sens chez Sénèque, Clem., I, 10, $3(8,3)$ : "tous les amants de sa fille, qui furent condamnés par lui pour ce crime, loin d'être mis à mort, reçurent de lui, avec l'ordre de partir, le sauf-conduit (diploma) qui achevait de les mettre en sécurité."
} 
afin de faciliter le voyage d'un officiel ou d'un chargé de mission. Cette clause ne concerne pas nécessairement les moyens de transport; elle peut porter sur la nourriture, le logement ou tout autre sorte de service ${ }^{98}$. Citons le cas de ce Vitalis, tabellarius, qui, muni d'un diplôme, a voyagé à pied"9 . Le diplôme n'est donc pas un passeport postal mais un "ordre de marche".

Sous la République, les diplomata étaient accordés par le Sénat en Italie et par les gouverneurs de province dans le reste de l'empire : ces derniers pouvaient les émettre pour les besoins de leur charge, sans référence à une autorité supérieure. Ce qui donnait lieu à des abus dont Cicéron se fait l'écho ${ }^{100}$. Sous le Principat, les diplômes sont émis par les empereurs, les préfets d'Egypte ${ }^{101}$, ainsi que par les gouverneurs des autres provinces et les consuls $^{102}$. C'est ce que prouve la fameuse inscription de Pisidie découverte à Burdur et analysée par S.Mitchell ${ }^{103}$. Daté de l'époque de Tibère, ce décret du légat propréteur Sextus Sotidius Strabo Libuscidiamus vise à réglementer l'usage du cursus publicus auprès de la cité de Sagalasus et à limiter les abus des officiels (et des soldats), qui tentent d'obtenir le transport sans payer (1.4) ou qui prennent plus qu'ils n'ont droit. L'inscription distingue précisément les gens qui ont le diploma provincial et les officiels venant d'autres provinces : à l'époque de Tibère, donc, les sauf-conduits proviennent encore de diverses sources. À partir de Domitien, en revanche, les diplomata ne sont émis que par l'empereur qui en donne un nombre limité aux gouverneurs de province. Cela se déduit notamment de la lettre de Domitien à son procurateur équestre de Syrie, Claudius Athenodorus, dans laquelle l'empereur ordonne de ne pas autoriser de réquisitions sur les provinciaux, sauf si le diploma a été émis par lui104. Cette situation nouvelle est confirmée par la correspondance de Pline : "les sauf-conduits dont le terme est expiré105 ne doivent pas être utilisés, lui écrit Trajan ; c'est pourquoi je tiens pour une de mes premières obligations d'envoyer dans toutes les provinces les nouveaux sauf-conduits avant qu'on puisse en avoir besoin"106. La réforme de Domitien est extrêment importante car elle tend non seulement à établir un contrôle, à limiter l'usage du cursus, mais à unifier l'émission des documents publics peut-être aussi à réduire le poids des recommandations privées de gouverneurs pour

\footnotetext{
${ }^{98}$ Comme l'a fait remarquer S.Mitchell (1976), c'est seulement à partir du IVe siècle que l'expression cursus publicus désigne exclusivement la poste impériale. Mitchell remarque ainsi que les principaux textes cités par H.G.Pflaum (1940) se réfèrent non à la poste impériale au sens strict, mais à la règlementation du transport d'officiels et de gens ayant une mission. Sur le cursus, voir en dernier lieu, E.W.Black, 1995, p. 4 sq.

${ }^{99}$ Cf CIL VIII, 1027 : diploma circavi totam regione $(m)$ pedestrem...Différents édits furent émis contre les excès commis par les détenteurs de ces diplomata (références plus bas, note 108).

100 Cf.Cic, in Pis., 90 où Cicéron accuse Pison, gouverneur de Macédoine, d'en distribuer trop facilement..

101 Cf. Tac., Hist., II, 65.

102 S.Mitchell, 1976 , p.126.

103 1976, p.125 sq, que nous suivons ici.

${ }^{104}$ IGLSyrie V, 1998 = A.MacCrum and A.G.Woodhead, 1966, n466 = P.F.Girard et F.Senn, 1977, p.435 sq.

105 Tous les sauf-conduits étaient datés et portaient le nom de l'empereur : aussi Caligula refusa-t-il ceux qui étaient datés de César ou de Auguste sous prétexte qu'ils étaient trop anciens (Suét., Cal., 38, 1) ; de même, ceux d'Othon étaient périmés sous Vitellius (Tac., Hist., II, 54, 1).

${ }^{106}$ Pline, Ep., X, 46, cf aussi X, 121.
} 
imposer celles de l'empereur. C'est ainsi précisément que s'exprimait Domitien (11.20 sq) : "car il est très injuste que l'influence personnelle ou le rang de quiconque permette les réquisitions que personne sauf moi-même n'a le droit d'autoriser".

Diverses inscriptions témoignent de la volonté de contrôler le cursus publicus ${ }^{107}$, mais le décret concernant Sagalasus présente l'intérêt d'indiquer très précisément les moyens employés pour mieux contrôler les réquisitions: "Puisqu'il y a des gens dont le manque de discipline suscite une punition, j’ai placé dans les cités et les villages une liste des prestations qui doivent être fournies (formulam eorum quae praestari indico in singulis civitatibus et vicis proposui)..." Le légat a aussi fait afficher la liste de ceux qui y ont droit (moyennant un paiement) : le procurateur et son fils en tête ${ }^{108}$, les sénateurs, les chevaliers, les centurions, les gens en service militaire... et les gens de son propre service qui bénéficient du logement et de repas. C'est dire qu'il fallait pouvoir prouver son appartenance à l'une de ces catégories.

Les réglementations strictes étaient nécessaires car les usurpations et les falsifications des diplômes étaient nombreuses. Elles semblent avoir été gravement réprimées au IIe siècle de notre ère ${ }^{109}$. On aimerait connaitre les formes que prenaient ces usurpations de comportement et surtout les contrôles d'identité qu'elles présupposaient (ou non), en amont. Mais les sources ne nous sont pas d'un grand secours.

\section{Les délégations étrangères}

Si les officiels romains détenaient des documents d'identification et si leurs déplacements donnaient lieu à un travail d'écriture, qu'en était-il des légations (étrangères ou provinciales) ? Par exemple, l'envoi d'ambassades de la part de pays belligérants était, à l'époque républicaine, contrôlé par le général chargé de la guerre : les sources littéraires et épigraphiques nous apprennent que ces derniers ne pouvaient se rendre à Rome sans une permission (permissum) et étaient accompagnés par des officiels romains ; qu'en cas de deditio, ils étaient même contraints d'y aller ${ }^{110}$, qu'ensuite, à Rome, ils devaient se présenter aux consuls pour ensuite être introduits auprès du Sénat ${ }^{111}$. Quant aux pays possédant un traité avec Rome, avaient-ils la liberté entière de s'y rendre?

\footnotetext{
${ }^{107}$ Citons par exemple l'édit du préfet d'Egypte Cn.Vergilius Capito sous le règne de Claude, cité par S.Mitchell, 1976, p. 126 (OGIS II, 665 (SEG VIII, 794); celui de Claude à Tégée en Arcadie en 49-50 : CIL III, 7251); ou encore l'édit de M.Petronius Mamertinus (133-137) prohibant les réquisitions des moyens de transport faites sans autorisation officielles (PSI V.446 = Hunt-Edgar; 1959-63, II, 221).

${ }^{108}$ La mention du fils (filioque eius) est toutefois contestée par B.Levick (1985, nº91, p.. 101-102) qui corrige en "son agent" (vilicoque eius).

109 D.48.10.27.2 (Modest. lib.8 regul.) : qui se pro milite gessit vel illicitis insignibus usus est vel falso diplomate vias commeavit, pro admissi qualitate gravissime puniendus est. Cf aussi CIG 4956 (inscription de la Grande Oasis, contre ceux qui ont fabriqué de faux diplomata).

${ }^{110}$ Exemple Liv., XLII, 36 : il est dit aux ambassadeurs de Persée qu'il “est inutile d'envoyer d'autres ambassades et que aucun d'entre eux n'aurait l'autorisation de traverser l'Italie" : nemini (...) eorum per Italiam ire liciturum. Pour d'autres sources, cf J.Linderski, 1995, p.465sq

111 Cf M.Bonnefond, 1989, p.294 sq. Sans doute à ce stade, il était procédé à une vérification de documents. On possède assez peu d'indices sur ces procédures concrètes de ce contrôle dont on a un écho dans le Digeste
} 
En ce qui concerne par ailleurs les légations des cités de l'empire, devaient-elles se procurer une autorisation, formelle ou écrite, du gouverneur de la province ? La lex Cornelia puis les mesures suivantes prises pour contrôler les dépenses des cités occasionnées par les ambassades $^{112}$ ne fournissent aucun indice concret. Mais Philon d'Alexandrie raconte que lors de l'arrivée de Caligula au pouvoir, les Juifs demandent au préfet Flaccus de transmettre leur décret de félicitation à Rome car "dans le cas où nous aurions sollicité une ambassade, il ne l'aurait pas autorisée"(oujk ajvn ejpevtreyen) ${ }^{113}$.

Il est vrai qu'Alexandrie n'est pas une cité grecque comme les autres ni l'Egypte une province ordinaire (nous en reparlerons). Mais il y a d'autres exemples et notamment le témoignage de Flavius Josèphe rapportant qu'après la dispute entre Festus et Agrippa II, les Juifs de Jérusalem demandèrent au procurateur Festus la permission d'envoyer une ambassade à Néron ${ }^{114}$; ou encore le décret des décurions de Sala (Mauritanie Tingitane) en 144, qui affiche la décision d'envoyer une députation au gouverneur pour lui demander la permission de mener une ambassade à l'empereur, dans le but de vanter l'action du préfet M.Sulpicius Felix. Voilà qui tendrait à prouver l'existence d'un contrôle effectif ${ }^{115}$. Mais de quand daterait sa mise en place ? Selon W.Williams, il s'agit d'une décision d'Antonin le Pieux : selon lui, avant ce règne, il n'existait pas de contrôle ou bien, autre hypothèse, seule une permission formelle était exigée ${ }^{116}$. La correspondance de Pline confirme négativement cette datation : elle nous montre un gouverneur de Bithynie qui, sous le règne de Trajan, ne contrôle pas les ambassades de Byzance et qui ne découvre leur fréquence qu'en examinant les comptes de la cité117. Mais la mesure d'Antonin n'aurait-elle pas eu de suite ? Pour les règnes de Marc Aurèle et de Commode, en effet, les sources ne fournissent aucun renseignement, et sous Septime Sévère, on constate un certain laxisme dans ce domaine. À partir de Constantin, en 317, les légations sont à nouveau soumises à l'accord du gouverneur mais on ne sait si cela fut maintenu après lui118.

\section{Les marchands}

Le déplacement des marchands était-il soumis à un contrôle autre que celui qui pesait sur les marchandises ${ }^{119}$ ? Dans une étude sur les marchands grecs au IVe siècle avant

(50.7.5, Scaevola, I regul.) : sed si non constat, legatus sit an non, Romae praetor de hoc cognoscerit. "S'il n'est pas suffisamment prouvé qu'il soit député ou non, le préteur de Rome doit en connaître"...

112 Fam., III., 10, 6 ; Dio, LVI, 25, 6 et Tac, Ann., XV, 22 : règlement augustéen ; D.L.7.5.6 : édit de Vespasien limitant à trois le nombre des ambassadeurs à chaque déplacement.

113 Flacc., 97 ; leg. ad Gai.., 247 : Petronius refuse l'ambassade des Juifs auprès de Caligula ; cf aussi Jos., Ant.Iud., XVII, 300 : unambassade juive arriva à Rome "sur l'autorisation donnée par Varus à la nation" ; cf aussi B.J.II, 80.

114 Ant.Jud., XX, 193-4 ; cf W.Williams,1967, p.475-77.

115 W.Williams, ibid. Le texte du décret de Sala est dans S.Gsell et J.Carcopino, 1931, 1-39 (:= AE 1931, 38) : deprecari(ve) prae/sidem indulgentissimum...permittat praeterea per legatos apud sacratissimum principem celebrare.

116 Ibid., note 44.

117 Ep., X., 43-44.

118 CTh, I.16.2 ; XII.1.9 ; cf F.Millar, 1977 p. 380.

119 Selon G.Pavis d'Escurac, “aucune entrave juridique ou administrative n'intervint jamais sous le Haut-

Empire, de la part des autorités de Rome ou de leurs représentants locaux, les gouverneurs de province, pour 
J.-C., Alain Bresson fait l'hypothèse que "les vérifications et contrôles effectués par les autorités portuaires dans un cadre juridique ordinaire ont dû permettre de déterminer la nature, la destination d'une cargaison, l'identité du commerçant"120. Peut-on en savoir plus ? Certaines catégories de navigants devaient nécessairement être en mesure de prouver leur statut: les negotiatores et les armateurs qui avaient passé des contrats avec l'État (à partir des mesures de Claude ${ }^{121}$ ) ou avec certaines cités et qui, à ce titre, jouissaient de privilèges ${ }^{122}$; ou bien la flotte d'Alexandrie, qui, selon G.Rickman, bénéficiait dans les ports italiens d'un traitement particulier ; ou encore les commerçants qui avaient reçu des autorisations d'exportations, comme ce fut le cas en 169 av.J.-C. pour les Rhodiens auxquels Rome accorda la faveur d'acheter 100000 médimnes de blé sicilien ${ }^{123}$; ou enfin les commerçants originaires d'un État avec lequel Rome avait passé un traité définissant des conditions commerciales avantageuses. Par exemple, les traités entre Carthage et Rome, retranscrits par Polybe, contiennent de nombreuses dispositions commerciales. Le premier définit deux zones, l'une de commerce libre, l'autre sous contrôle public (assumé par des fonctionnaires — hérault et grammateus - pour surveiller et garantir les échanges entre Carthaginois et Romains) (III, 22, 8) ; il est encore précisé que "les Carthaginois ne feront aucun tort au peuple d'Ardée, d'Antium, de Laurente, de Circé, de Terracine, ni à aucun autre des peuples latins qui sont soumis à Rome” (III, 22, 11). Le deuxième traité prévoit qu’il sera possible aux Carthaginois de venir commercer à Rome (III, 24, 11). Ces catégories privilégiées devaient donc posséder des titres leur permettant de faire respecter leurs droits et, bien que les sources ne comportent pas d'indication précise, on peut penser, en raison du caractère officiel de ces échanges, aux symbola, bien connus dans le monde grec, et plus généralement aux sceaux ${ }^{124}$.

Mais qu'en était-il du marchand ordinaire qui devait déclarer ses marchandises à tous les portoria ou même du voyageur qui passait sur les routes à péages ?

De nombreuses études, à commencer par celle de S.J.De Laet, ont montré que les portoria étaient soit des droits de douanes, soit des octrois soit de simples péages aux

\footnotetext{
surveiller, vérifier ou limiter les déplacements des marchands. Les exigences du portorium n'imposaient qu'aux seules marchandises les contrôles et les taxes que nous savons dans les stationes douanières [...] L'empire est bien une unité territoriale à l'intérieur de laquelle circulent, au gré de leurs projets commerciaux, tous ceux qui veulent et savent profiter des opportunités économiques"(1988, p.57). Elle rappelle la dissertation de V.Parvan, 1909, p. 126, selon qui, l'empire a connu la libre circulations des marchands. Ces affirmations bien complexes mériteraient cependant de plus vastes enquêtes.

120 1977, p. 60 ; cf aussi D.van Berchem, 1991.

121 Suet., Claud., 18.2-19.1 ; cf aussi Gaius, Instit., I.32 C ; D.50.4.5 ; 5.3; 6.6.3 sq.

122 Selon G.Rickman, 1980, qui s'appuie sur D.50.5.10.1 ; 50.6.6.5, ils doivent donc disposer de contrats attestant leurs fonctions et leurs droits afférents, que ce soit avec ou sans la collaboration des collegia auxquels ils appartenaient et qui, selon Rickman, pouvaient jouer un rôle dans l'administration des ports ; cf aussi G.W.Houston, 1980 ; le problème se pose de la même façon pour les mancipes qui prennent en charge le blé de Sicile, C.Nicolet, 1991.

123 Polybe, Hist., 28,2 : des ambassadeurs rhodiens étaient venus à Rome "pour renouveler le traité d'amitié et demander l'autorisation de faire des achats de blé" (sivtou qevlonte" ejxagwgh;n labei'n).

${ }^{124}$ Sur les traités entre Rome et Carthage, voir B.Scardigli, 1991, p.73 sq ; sur les symbola, cf Ph.Gautier,1972.
} 
frontières de l'empire ou à l'intérieur ${ }^{125}$. Arrivés à ces postes, les voyageurs devaient déclarer leurs marchandises, même s'ils jouissaient d'exemptions ${ }^{126}$. Alors, ils recevaient un justificatif, tandis que les agents du portorium inscrivaient la déclaration sur des tabulae. Si les voyageurs étaient convaincus de fausse déclaration, après une fouille par exemple ${ }^{127}$, les marchandises étaient confisquées (cadere in commissum) mais les moins de vingt cinq ans échappaient au commissum ${ }^{128}$. La fouille était sans aucun doute l'objet d'abus, et les sources y font allusion dès les époques anciennes. Le portitor ou douanier pouvait en effet tout fouiller, y compris les registres des marchands, et prendre de connaissance de tous les documents cachetés ${ }^{129}$.

On possède un exemple littéraire d'une de ces déclarations dans la vie d'Apollonius de Tyane de Philostrate (Vita Ap.I,20) : le philosophe se rendant en Mésopotamie par Zeugma se voit questionné par le douanier (telwvnh") qui le conduit devant le pinavkion (tableau d'affichage des tarifs douaniers ${ }^{130}$ ) et lui demande ce qu'il a à déclarer : Apollonius doit remplir de sa main un registre sur lequel il inscrit seulement la liste de ses biens (y compris les esclaves). Il ne lui est apparemment rien demandé sur son identité131. . En tout cas, nos sources sont muettes sur ce point.

Le cas particulier des exemptions jette cependant quelque lumière sur la question. Prenons en exemple les marchandises qui, destinées au personnel militaire et administratif, n'étaient pas soumises à des taxes : toute personne chargée de leur transport devait "signer un libellus de sa propre main et l'adresser au responsable des douanes (publicanus)"132. Ce dernier ne taxait donc que le surplus. On imagine aisément tout le travail d'écriture qui était accompli pour contrôler les marchandises et vérifier l'identité du marchand. Il en était de même pour les particuliers bénéficiaires de l'immunitas en matière de douane, tel le sophiste Polémon $^{133}$ : Trajan lui concéda le privilège de voyager gratuitement (ajtelh` poreuvesqai)

\footnotetext{
125 S.J.De Laet, 1949 ; S.E.Sidebotham, 1986 avec la bibliographie plus récente sur le sujet. Il faut compter aussi désormais avec l'inscription d'Ephèse : cf H.Engelman et D.Knibbe, 1989 ; C.Nicolet, 1990 ; 1991 ; 1993 ; 1999 ; T.Spagnuolo-Vigorita, 1997. Les tarifs des douanes variaient selon qu'elles étaient impériales ou municipales : cf l'intéressant décret de Caunos qui rappelle les conditions dans lesquelles seront exemptés les seuls habitants de Caunos (citoyens, métèques et étrangers résidents) et l'analyse de G.E.Bean, 1954, p. 7 sq. 126 Cf Inscription d'Ephèse (éd.Engelman et Knibbe, 1989), \22, 11.53-56 : interdiction d'importer ou d'exporter ce qui n'a pas été déclaré par écrit (ajnapovgrafon); pour une interprétation de ce passage, voir T.Spagnuolo-Vigorita, 1997, p.173.

127 Cf Cic., de leg. agr., II, 23, 61 ; In Vat., 12 ; Plut., de curios., 7 ; et surtout Quint., Declam., 341 qui présente un dialogue fictif entre un douanier et un particulier dépouillé de ses biens après une fouille.

128 D.IV.4.9.5 : on se demandera ici sans insister comment se faisait l'attestation de minorité. Sur les conditions du commissum, cf D.XXXIX, 4, 11, 4-6 surtout. Cf aussi les règlements visant à protéger les voyageurs contre les exactions fiscales (ex : P.Princ.II, 20, col I).

129 Plaut., Trin., 770-819 ; Tér., Phorm., 149-150.

130 On connaît plusieurs de ces affichages, par exemple le tarif de Zarai, daté de 202 (CIL VIII 4508) ou le tarif de Palmyre (cf S.J.De Laet, 1949, p. 356 sq ; J.F.Matthews, 1984 ; J Teixidor, 1983)

131 Même chose sur le territoire parthe (I,21), où, approchant de Babylone, Apollonius rencontre une garnison du roi. Le satrape qui la commande, lui demande de déclarer qui il est, de quelle cité il vient et les raisons de ce voyage.

132 D.39.4.4.1 (Paul) : significet libello manu sua subscripto, erumque ad publicanum mittat (et C.Nicolet, 1999, p.201

sq) ; cf aussi C.Th.XIII $8.1 ; 5.33$ (et S.Mitchell, 1976).

133 Philostr., Vit.Soph., II, 25 (Polemon de Laodicée, p. 44.3, éd.Kayser, Teubner).
} 
"par terre et par mer"134 ; Hadrien étendit ce bénéfice à ses descendants. Polémon était donc exempté de tout portorium. Mais comment justifiait-il son exemption et comment ses fils le faisaient-ils? La même question se pose pour les citoyens romains qui par traité ont reçu la franchise dans un port qui garde le droit de lever des taxes ${ }^{135}$.

Lorsqu'un particulier avait reçu un privilège impérial, il lui fallait, pour le faire reconnaitre dans sa cité, le déclarer publiquement, confirmation impériale à l'appui, et aussi se soumettre à une vérification d'aptitude comme le laisse entendre Sénèque ; le privilège était ensuite enregistré dans les archives locales ${ }^{136}$. Mais sur les routes ? Il fallait bien que les exemptés aient détenu un document d'attestation, tout comme les vétérans de l'armée détenaient depuis Claude une copie du diplôme militaire, sorte de document d'identité et d'immunité ${ }^{137}$. Les beneficia, catégorie dans laquelle rentrait l'immunitas, enregistrés dans des libri beneficiorum à Rome ${ }^{138}$, donnaient lieu en effet à la délivrance au bénéficiaire d'un libellus, copie de la décision impériale attestant son enregistrement dans les archives ${ }^{139}$. L'existence de ces documents d'exemption permet de lever un coin de voile sur les contrôles d'identité aux postes de douanes.

Au contrôle des douanes il faudrait enfin ajouter les contrôles routiers. Nous ne citerons ici que le tarif de Coptos, de l'époque de Domitien ${ }^{140}$, ensemble des droits à payer pour l'usage de la route qui menait de Coptos à l'un des ports de la mer Rouge. Rien à voir ici avec un contrôle douanier : les passants ne déclaraient pas leurs marchandises mais acquittaient un droit de passage. La route était entretenue aux frais du gouvernement, des

\footnotetext{
134 Sur le caractère technique de cette expression, cf les exemples cités par J.France, 1999, p. 104 sq.

135 Liv., 38, 44, 4 (189 av.J.C.) : selon le décret concernant la liberté d'Ambracie, les gens d'Ambracie pourraient "lever les droits de douane qu'ils voudraient pourvu qu'en fussent exemptés les Romains et leurs alliés latins" ; cf décret d'Auguste pour Séleucos de Rhosos (1l.45-50) : FIR A I, n.55.

136 Sénèque, Ben., IV, 28,5 : "si tu exiges ...une appréciation des personnes (aestimationem personarum), que ce soit pour les dons individuels qui s’adressent spécialement au mérite" ; cf les très intéressantes remarques dans N.Lewis, 1966, notamment p.515 sq, qui se demande par quel moyen, un médecin, par exemple, pouvait revendiquer les exemptions auxquelles sa profession donna droit à certaines époques. Il relève d'une part que tous les médecins n'étaient pas exemptés systématiquement; que d'autre part, il fallait une confirmation nominale (impériale, provinciale, municipale) fondée sur une docimasie (vérification d'aptitude civique et pratique : cf C.J.X.3.1 ; P.Oxy., 40) et l'on peut supposer ainsi que cette docimasie était suivie d'un enregistrement dans les archives locales. À rapprocher de D.50.6.6.7 : si un individu est appelé à assumer des munera avant d'avoir [...] déclaré publiquement son immunité (antequam publice profiteretur) [...], il devra les assumer."

137 Aux vétérans, l'immunité n'a pas toujours été accordée. mais c'est le cas par exemple avec le décret de Domitien qui a donné aux vétérans et à leurs familles l'exemption des taxes douanières : omnibus vectigalibus portoribus liberati (AE 1910, 75 = CIL XVI, p. 146, n.12); Là aussi il faut un document nominal. Cf BGU III, 812 : le document désigne un certain Festus, immune; selon S.L.Wallace, il s'agit précisément d'un vétéran bénéficiant de l'exemption grâce à la mesure de Domitien (1938, p.267).

138 Cf C.Moatti, 1993, p. 56sq.

139 Cf W.Seston, 1971 ; pour les terres, C.Moatti, 1993, p. 56-61. Une procédure proche de l'insinuatio, instituée par Trajan, qui consiste à envoyer l'original d'un rescrit au petitor. Cf G.Cencetti, 1953, p. 164 ; sur les différents sens donnés au terme insinuatio, cf N.Palazzolo, 1977, p.43 et p.87.

140 IGRRom.I, 1183 = OGIS 674 = Mac Crum and Whoodhead, $\mathrm{n}^{\circ} 459$ (90 ap.J.C.) ; cf S.L.Wallace, 1938, p. 273-74 ; S.J.De Laet, 1949, p.329-30. D’autres tarifs sont cités dans S.E.Sydebotham, 1986, p.35 sq.
} 
stations (mansiones) y étaient disposées à intervalles réguliers pour le ravitaillement en eau ${ }^{141}$ ; c'était donc une route officielle, mais ouverte aux civils. Un permis écrit (pittavkion) était délivré142 mais c'était seulement une attestation de péage. Toutefois, la somme à payer variait non seulement selon le moyen de transport utilisé mais, ce qui nous intéresse ici, selon la qualité des personnes : par exemple, les femmes de soldats payaient 20 drachmes, mais les prostituées 108. Là encore on se demandera si la professio ou l'“uniforme" constituaient des indices suffisants.

\section{Le cas de l'Egypte :}

Le tarif de Coptos nous introduit dans un univers apparemment beaucoup mieux connu, celui de l'Egypte. Dans cette province si particulière, le contrôle des personnes, déjà bien attesté sous les Ptolémées, restait important à l'époque romaine : Strabon témoigne qu'“il n'était pas possible sans ordre de mission (ajuvneu prostavgmato") de sortir d'Alexandrie par mer... ni de s'échapper par mer en secret étant donné l'importance de la garde qui fermait le port et toutes les issues. Nous avons pu le constater par nous-même, pour ce qui en subsiste encore aujourd'hui, pendant l'assez long séjour que nous avons fait à Alexandrie, quoique de nos jours, avec la domination romaine, le contrôle se soit relâché"143. Un contrôle qui s'explique en grande partie par le fait que "chaque individu était considéré comme une source présente ou potentielle de revenus"144. Mais le texte de Strabon est peu précis. Qui devait se procurer l'ordre de mission et sous quelle forme se présentait ce document ? Qui le délivrait ? En avait-on besoin pour entrer et pour sortir ? Il importe donc de reprendre le dossier et d'en reconsidérer les termes.

On dispose en réalité de plusieurs sources importantes. Tout d'abord quelques chapitres du Gnomon de l'Idiologue, qui résume les règlements de ce "département des revenus spéciaux", rédigés sous le règne d'Antonin le Pieux, mais comportant une expérience accumulée sur 200 ans ${ }^{145}$. Article 64 : "les poursuites contre les personnes qui se sont embarquées (à Alexandrie) sans "document officiel” (cwri;" ajpostovlou) relèvent actuellement de la juridiction du préfet." Article 66 : "les personnes à qui il a été permis d'embarquer mais qui sont parties sans "document officiel” (ajnapovstoloi), sont punies de

\footnotetext{
141 Pline, NH., VI, 102 ; pour d'autres exemples, cf B.Isaac, 1992, p. 200 sq ; voir aussi, pour le IVe siècle, le très intéressant témoignage du voyage d'Egérie 9,2-3; Egérie témoigne aussi que sur certains tronçons de la route, des soldats accompagnaient les pèlerins pour les protéger (cf E.D.Hunt, 1984, p.59).

142 Pittakion désigne clairement un document écrit, volumen ou tablette (Polyb., 31, 13, 9), mais ce document peut avoir différents usages : cf Liddle Scott, s.v.pittakion ; cf A.D’Ors, 1951.

143 Géogr., II, 3, 5 , 101. En réalité il était possible d'accoster à Alexandrie sans document et d'échapper au contrôle : Philon d'Alexandrie (Flacc., 27-28) raconte qu'Agrippa, fils d'Hérode, parti de Rome pour Alexandrie, y accosta à l'insu de tous et y séjourne secrètement; même chose dans l'affaire du centurion Bassus ( $\left.\int 97\right)$.

144 O.W.Reinmuth, 1935.

${ }^{145}$ Le texte est connu par deux papyri : BGUV, 1210 et P.Oxy. XLII, 3014. La traduction et les premiers commentaires sont dus à T.Reinach, 1919-1920 (cf aussi Girard, I, p.OOO-. Après lui, la bibliographie est importante sans être massive : cf notamment P.R.Swarney, 1970 ; Cf S.Riccobono, 1950, p.203; cf R.Taubenschlag II, 1948, p. 62-63.
} 
la confiscation du tiers de leurs biens. Si elles ont fait sortir sans document (ajnapostovlou") leurs propres esclaves, la confiscation est totale"146.

Selon T.Reinach, apostolon est l'équivalent de prostagma $^{147}$. Mais le mot prostagma désigne tout simplement une ordonnance royale ${ }^{148}$; il ne nous est donc pas très utile pour définir la nature du document. Plus intéressant est le rapprochement avec ejpivstolh ${ }^{149}$, document envoyé par les officiels du port aux armateurs et capitaines de navire possédant un chargement de blé : c'est en quelque sorte un ordre de navigation dans lesquels les marchandises ont autant d'importance que les personnes. Ce sens est renforcé par un rapprochement avec P.Oxy. 1259 dans lequel apostolos est clairement "a custom certificate drawn up by the procurator, consisted in an identity card for a sailor and instructions to local authorities concerning a grain shipment" ${ }^{150}$.

L'article 68 du Gnomon, d'autre part, évoque le cas des Romains contraints de présenter des “documents” pour quitter l'Egypte. Mais les mots pour les désigner diffèrent des articles précédents : "un Romain qui s’embarqua sans avoir réuni au complet les pièces exigées pour la sortie (ta; pro;" ejkplou `n gravmmata) fut condamné à une amende de (tant de) talents". Or on peut rapprocher ce passage de deux documents : d'une part du P.Oxy. 1260 où le terme grammata renvoie aussi à des instructions de navigation ${ }^{151}$ et d'autre part d'une lettre de Cicéron à Atticus datée de 49 dans laquelle Cicéron révèle que son ami a reçu un sauf-conduit (ce qu'il appelle ailleurs un diploma) pour traverser l'Adriatique et rejoindre l'Épire. Le mot que Cicéron emploie, nous l'avons vu ${ }^{152}$, est, d'après la meilleure restitution, ejkplou `n, mot qui désigne à la fois la sortie d'un port et le droit de sortie ${ }^{153}$. On pourrait donc comprendre que, si l'exportation des marchandises nécessitait d'un ordre de mission (ajpovstolon), les personnes devaient se procurer un sauf-conduit, une sorte de permis de circuler nominal, un diploma. Cette distinction pourrait être confirmée par P.Oxy. X.1271 (daté de 246) : À Valerius Firmus, préfet d'Egypte, de la part d'Aurelia

\footnotetext{
146 D'autres articles précisent le sort des personnes ayant négligé la loi : l'article 69 rappelle qu'une Egyptienne qui fit sortir par Péluse des esclaves (avec la complicité de ses fils ?) fut condamnée à une amende d'un talent et 3000 drachmes ; l'article 65 que des esclaves exportés par leur maittre ignorant (de la loi) ont été vendus aux enchères ; l'article 67 que "les personnes qui ont hypothéqué (?) ou vendu des enfants nés dans leur maison d'esclaves égyptiens sans présenter l'acte de naissance (?) afin de les faire sortir du pays, ont été punies tantôt de la confiscation totale, tantôt de celle de la moitié ou du quart."

147 1920, p.119.

148 Cf par exemple M.T.Lenger, 1952, p.222 sq.

149 T.Reekman et E.Van't Dick, 1952, p.179.

150 Cf S.E.Sidebotham, 1986, p.80 : le P.Oxy. 1259 reproduit le reçu de marchandise : "À Didyme, stratège du nome d'Oxyrhinchos, de la part de Posidonios, aussi appelé Triadelphus, maître de 8 bateaux emportant 40 000 artabae de l'administration de Neapolis. J'ai reçu ... le montant que vous m'avez commandé, vous et Horion, alias Apion, en accord avec le message de son excellence le procurateur de Neapolis (ejx ajpostovlou tou' krativstou th " Neva" polevw"'. L'apostolon est bien un ordre de navigation nominal pour le transport de marchandises.

151 1.7 : "J'ai reçu et embarqué sur mon bateau d'après les instructions d’Ulpius Cyrillus (ejk gravmmata Oujlpivou Kurivllou)..."

152 Plus haut, note 93.

153 Liddel-Scott, I, p. 517 ; cf aussi IG 12 (7).8.12 (Amorgos) et GDI 5687.8 (Chios) où ejkplou’n est employé au sens de "droit de sortie".
} 
Maeciana de Side ${ }^{154}$. Je voudrais, maître, prendre le bateau à Pharos. Aussi je vous prie d'écrire au procurateur de Pharos de me laisser partir, comme c'est l'habitude (bouvleumai, Kuvrie, ejkpleu`sai di;a Favrou: Ajxiw` gravyai se tw “ú ejpitrovpw th " Favrou ajpolusaiv me kata; to; ejvqo"). Adieu"155. Suit la subscriptio du préfet. Il semble bien que l'on ait dans ces textes les mêmes mots que dans l'article 68 du Gnomon. Ainsi, la distinction entre les documents ne semble pas avoir été liée au statut de celui qui voulait quitter l'Égypte ${ }^{156}$ mais à la nature de la navigation. Dans un cas, ce sont des documents d'identité (diploma, traduit ici par ta; pro;" ejkplou `n gravmmata), dans l'autre des documents fiscaux (apostolon). Mais l'un comme l'autre désignent des autorisations de circuler, de se déplacer.

Quelle que soit la nature du document, il fallait réclamer l'autorisation de sortie au préfet qui remplace l'Idiologue à l'époque de rédaction du Gnomon. Le préfet l'endossait et l'envoyait au procurateur du port de départ qui exerçait les fonctions d'inspecteur de l'émigration et de collecteur de taxes sur les marchandises. Certains historiens se sont demandé si, dans la mesure où les délits relevaient de l'Idiologue, ce document était payant $^{157}$ Ils ont en effet rapproché à juste titre le permis du Gnomon du tarif de Coptos. Dans les deux cas, le permis se rapproche d'un péage et bénéficie au gouvernement ; dans les deux cas, l'aspect fiscal est indissociable du contrôle des personnes elles-mêmes. Mais les sources ne disent rien d'explicite à ce sujet.

Il existait ainsi une multitude de cas particuliers qui nécessitaient des moyens de contrôle soit pour des mesures de surveillance soit pour des raisons fiscales ou économiques, soit pour faire respecter des droits. Ce sont tous ces aspects que nous avons voulu souligner même si l'enquête présente de nombreuses lacunes. Il nous a paru nécessaire en tout cas de tenter l'épreuve et puisqu'il est question de mobilité, d'ouvrir des pistes.

Claudia Moatti

Université de Paris 8- Saint-Denis-Vincennes

154 En Pamphylie.

155 Cf le commentaire de P.J.Sijpesteijn, 1981, p.108-109 concernant la date du papyrus

156 Contr. S.E.Sidebotham, 1986, p.80.

157 S.L.Wallace, 1933, p.273 sq ; suivi, par exemple, par S.E.Sidebotham, 1986, p.80-81. 


\section{BIBLIOGRAPHIE}

G.Amiotti, Primi casi di relegazione e di deportazione insulare nel mondo romano, dans M.Sordi éd., Coercizione e mobilità umana nel mondo antico, Milan, 1995, p.

R.Andreotti, Su alcuni problemi del rapporto fra politica, sicurezza e controllo del commercio nell' impero romano, dans $R I D A, 16,1969$, p.215-257

N.J.E.Austin et N.B.Rankov, Exploratio. Military and Political Intelligence in the Roman World from the second Punic War to the battle of Andrinople, Londres et NewYork, 1995

J.P.V.D.Baldson, Romans and aliens, Londres, 1979

J.Barbeyrac, Histoire des anciens traités ou Recueil historique et chronologique des traités répandus dans les auteurs grecs et latins et autres monuments de l'antiquité jusqu'à Charlemagne, La Haye, 1739

G.E.Bean, Notes and Inscriptions from Caunus, dans JHS, 74, 1954, p. 97 sq

E.Benveniste, Notes étrusques. I- La tablette d'ivoire deCarthage, dans SE, 7, 1933, p.245-49

D.van Berchem, Commerce et écriture, dans Museum Helveticum, 48, 1991, 3, p.129-145

E.J.Bickerman, Studies in Jewish and Christian History, I-II, Leiden, 1980

E.W.Black, Cursus publicus. The infrastructure of Government in Roman Britain, BAR British Series 241, 1995

A.L.Boegehold, The Establishment of a Central Archive at Athens, dans AJA 76, 1972

M.Bonnefond-Coudry, Le sénat de la république romaine, Rome, BEFAR, 1989

Ph.Boutry et D.Julia éd., Pèlerins et pèlerinage dans l'Europe moderne, Actes de la table-ronde organisée à Rome les 4-5 juin 1993, Rome, 2000

A.Bresson, L'attentat d'Hiéron et le commerce grec, dans Les échanges dans l'Antiquité : le rôle de l'Etat. Entretiens de Saint Bertrand de Comminges, 1994, p. 47-68

F.Burkhalter et A.Martin, Microcosmes et macrocosmes : la segmentarisation des populations. Le cas d'Alexandrie, dans C.Nicolet éd., 2000, p.253-279

L.Canfora, M.Liverani, C.Zaccagnini, I trattati nel mondo romano : forma, ideologia, funzione, Rome, 1990

J.Carcopino et S.Gsell, La base de M.Sulpicius Felix et le décret des décurions de Sala, dans MEFR.XLVIII, 1931, 1-39

J.M.Carrié, Les distributions alimentaires dans les cités de l'Empire romain tardif, dans MEFRA, 87, 1975, 2, p.995-1101

L.Casarico, Il controllo della populazione nell'egitto romano, 1-Le denunce di morte, Arrate, 1985

L. Casson, Ships and Seamanships in the Ancient World, Princeton, 1971 ; Travel in the Ancient World, Londres, 1974

S.Cataldi, Symbolai e relazioni tra le città greche nel V sec.a.C., Pise, 1983

G.Cencetti, Tabularium principis, dans Studi Manaresi, Milan, 1953, p.131-166

V.Chapot, La frontière de l'Euphrate de Pompée à la conquête arabe, Paris, BEFAR 99, 1907

A.Chastagnol, La préfecture urbaine à Rome sous le Bas-Empire, Paris, PUF, 1960

A.Chastagnol, Le problème du domicile légal des sénateurs à l'époque impériale, dans Mélanges Senghor, 1977, p. 43-54 
J.Chelini et al., Histoire des pèlerinages non chrétiens, Paris, 1987

M.R.Cimma, Reges socii et amici populi romani, Milan, 1976

F.Coarelli, I monumenti dei culti orientali a Roma.Questione topografiche e cronologiche, dans La soteriologia dei culti orientali nel mondo romano, Leyde-Rome, 1982, p.33-66,

M.Corbier, Cité, territoire et fiscalité, dans Epigrafia. Actes du colloque en mémoire de Attilio Degrassi, Rome, 22-28 mai 1988, Rome, 1991, p.629-655

F.Cordano, Le tessere pubbliche dal tempio di Athena a Camarina, Rome, 1992

L.Cracco Ruggini, Fame Laborasse Italiam : una nuova testimonianza sulla carestia del 383 d.C., dans Ath. 1976, vol.Sp. Studi P.Fraccaro, p.8398

L.Cracco Ruggini, Nuclei immigrati e forze indigene in tre grandi centri commerciali dell'impero, dans J.D’Arms et E.C.Kopff éd., The Seaborne Commerce of Ancient Rome, 1980, p.55-76

L.Cracco Ruggini, Spazi urbani clientelari e caritativi, dans Rome impériale, démographie et logistique, Rome, Coll E.Fr.R. 230, 1997, p.157-191

G.Crifò, Ricerche sull'exilium, Rome, 1971

J.D'Arms et E.C.Kopff, The Seaborne Commerce of Ancient Rome. Studies in Archaeology and History, Rome, M.AAR, 36, 1980

E.Deniaux, Clientèles et pouvoir à l'époque de Cicéron, Rome, Coll.EFrR n¹82, 1993

S.J.De Laet, Portorium. Etudes sur l'organisation douanière chez les Romains, surtout à l'époque du Haut-Empire, Bruges, 1949

A.D’Ors, Pittavkion - pittaciarium, dans Aegyptus, 1951, p.339-343

H.Engelmann et D.Knibbe, Das Zollgesetz der provinz Asia. Eine neue Inschrift von Ephesos, Bonn, 1989

H.van Effenterre, Le statut comparé des travailleurs étrangers à Chypre, en Crète et autres lieux de l'archaïme, dans Acts of the International Archaeological Symposium. The Relations between Cyprus and Crete ca 2000-500 B.C. (Nicosia, 1978), Nicosie, 1979, p. 279-293 = Cretica selecta, 1990, p.647-662

E.Faure, Saint Ambroise et l'expulsion des pérégrins de Rome, dans Mélanges G.Le Bras, 1965, I, p.523-540

J.L.Ferrary, Traités et domination romaine dans le monde hellénistique, dans L.Canfora et al., 1990, p.217-235

J.France, Les revenus douaniers des communautés, dans Il capitolo delle entrate nelle finanze municipali in occidente ed in oriente, Actes de la Xe Rencontre franco-italienne sur l'Epigraphie du monde romain (Rome, 27-29 mai 1996), Coll.E.Fr.R., Rome, 1999, p.95-109

J.Gagé, Les classes sociales dans l'Empire romain, Paris (1964), 1971

J.Gaudemet, L'étranger au Bas-Empire, dans L'étranger, Recueils de la société J.Bodin, 9, 1958, p.209-235

Ph. Gauthier, Symbola. Les étrangers et la justice dans les cités grecques, Nancy, 1972

Ph.Gauthier, Les cités grecques et leurs bienfaiteurs (IV e s-Ier avJC). Contribution à l'bistoire des institutions, dans BCH.Suppl.XII, Paris, 1985 
A.Giardina, Aspetti della burocrazia nel Basso Impero, Rome, 1977

P.F.Girard et F.Senn, Textes de droit romain. II - Les lois des Romains, 7e éd. tome 2, Naples, 1977

W.Goffart, Barbarians and Romans, The techniques of accomodation, AD418-584, Princeton

U.Press, 1980

R.G.Goodchild, The limes Tripolitanus II, dans JRS 40, 1950, p. 30 sq

R.Gordon, dans R.Gordon et al., Roman Inscriptions 1986-90, dans JRS, 83, 1993, p.155-6

M.Gras, Trafics tyrrhéniens archaïques, BEFAR 258, Rome, 1985

F.Grelle, Città e trattati nel sistema romano imperiale, dans L.Canfora, 1990, p. 237-256

S.Gsell et Carcopino, La base de M.Sulpicius Felix et le décret des décurions de Sala, dans

MEFR.XLVIII, 1931, 1-39

J.Hatzfeld, Les trafiquants italiens dans l'Orient hellénique, Paris, 1919

G.W.Houston, The administration of the Roman Ports, dans J.D’Arms et E.C.Kopff éd, 1980, 157-171

A.S.Hunt et C.C.Edgar, Select Papyri, Londres, Cambridge (Mass.), 1959-1963

E.D.Hunt, Holy Land Pilgrimage in the Later Roman Empire, AD 312-460, Clarendon

Press,Oxford, 1984

B.Isaac, The limits of Empire, The Roman Army and the East, Cambridge (1990) 2e édition 1992

P.Jouguet, La vie municipale en Egypte romaine, Paris, 1911

D.Julia, Pour une géographie européenne du pèlerinage, dans Ph.Boutry et D.Julia éd., 2000, p.3126

J.Juster, Les Juifs dans l'empire romain, Paris, 1914

G.La Piana, Foreign Groups in Rome, dans Harvard Theological Review 20, 1927, p.183-403

J.M.Lassère, Ubique populus. Peuplement et mowvements de population dans l'Afrique romaine de la chute de Carthage à la fin de la dynastie des sévères (146-235 ap), Paris, 1977

M. Lauria, Il gnomon dell'idios Logos", dans Atti Accad.sc.mor.e pol., Naples, 75, 1964, p. 1 75

M.T.Lenger, Un corpus de la législation ptolémaïque, dans C.E.27, 1952,p.218-246

M.A.Levi, L'esclusione dei senatori romani dall'Egitto Augusteo, dans Aegyptus, 5, 1924, 231-35

B.Levick, The government of the Roman Empire. A sourcebook, Londres, 1985

N.Lewis, Exemption from Liturgy in Graeco-roman Egypt, dans Actes du Xe Congrès intern. des

Papyrologues, 1964 p.69-78 et dans Atti dell'XI Congresso internazionale di Papirologia, 1966, p.508-541 = The Compulsory Public Services of Roman Egypt, Papyrologia Florentina XXVIII, Florence, 19972

J.Linderski, "Ambassadors go to Rome", dans E.Frézouls et A.Jacquemin éd., Les relations internationales, Actes du colloque de Strasbourg (15-17 juin 1993), Paris, 1995, p.453-478

A. Lintott, Imperium romanum. Politics and administration, Londres et New York, 1993

A.MacCrum et A.G.Woodhead, Select Documents of the Principate of the Flavian Emperors including the year of Revolution A.D.68-96, Cambridge, 1961 
R.MacMullen, Soldier and Civilian in the Later Roman Empire, Cambridge, Mass., 1963

D.Magie, Roman rule in Asia Minor, Princeton, 1959

G.Mancini, Cives romani Municipes Latini, Naples, 1996

J.C.Mann et R.R.Roxan, Discharge Certificates of the Roman Army, dans Britannia, XIX, 1988, p.341-347

H.I.Marrou, Histoire de l'éducation dans l'antiquité, (1948), t.2 : Le monde romain, Points, Seuil, Paris, 1981

M.R.Marrus, Les exclus. Les réfugiés européens au XIXe s., Paris, Calman Levy, 1986 (1ère éd.1985)

J.F.Matthews, The Tax Law of Palmyra, dans JRS, 74, 1984, p.157-180

Mathisen, Emigrants, exiles and survivors : aristocratic options in Visigothic Aquitania, dans Phoenix, 38, 1984, p.159-170

S.Mazzarino, Aspetti sociali del quarto secolo. Ricerche di storia tardo-romana, Rome, 1951

F.Millar, Emperors, frontiers and foreign relations, 31 B.C to A.D.378, dans Britannia, 1982

F.Millar, The Emperor in the Roman World, Londres, 1977

S.Mitchell, Requisitioned Transport in the R. Empire. A New Inscription from Pisidia, dans JRS, 66, 1976, p. 106-131

L.Moretti, Sulle stationes municipiorum del foro romano, dans Athenaeum, XXXVI, 1958, 106116

J.Napoli, Recherches sur les fortifications linéaires romaines, Coll. Ecole française de Rome 229, Rome, 1997

V.Neri, I marginali nell'occidente tardoantico. Poveri, infames e criminali nella nascente società cristiana, Edipuglia, Bari, 1998

C.Nicolet, Le Monumentum Ephesenum, la loi Terentia-Cassia et les dîmes d'Asie, dans MEFRA, 111, 1999, 1, p.191-215

C.Nicolet, Frumentum mancipale : en Sicile et ailleurs, dans A.Giovannini éd., Nourrir la plèbe. Actes du colloque de Genève, 28-29 IX.1989, Hommage à D.van Berchem, Bâle-Kassel, 1991, p.119-141

C.Nicolet, À propos du règlement douanier d'Asie : démosiôna et les prétendus quinque publica Asiae, dans $C R A I, 1990$, p.675 sq

C.Nicolet, Le monumentum Ephesenum et les dîmes d'Asie, dans BCH 115, 1991, p.465 sq

C.Nicolet, Le monumentum Ephesenum et la délimitation du portorium d'Asie, dans MEFRA 105, 1993, P. 929 sq

C.Nicolet éd., Mégapoles méditerranéennes. Géographie urbaine rétrospective, Maisonneuve et

Larose, Maison méditerranéenne des sciences de l'homme, Ecole françasie de Rome, 2000

W.Nippel, Policing Rome, dans JRS, 74, 1984, 20 sq

W.Nippel, Aufrubr und Polizęi in der röm.Republik, Stuttgart, 1988 (tr. angl. Police at Rome, 1997)

G.Noiriel, Le creuset français. Histoire de l'immigration XIXe-XXe s., Paris, Seuil, 1988 
G.Noiriel, Surveiller les déplacements ou identifier les personnes??, dans Genèses, 30, mars 1998, p. 77-100

D.Nordman, Sauf-conduits et passeports en France, dans J.Céard et J.C.Margolin éd, Voyager à la renaissance. Actes du colloque de Tours 1983, Paris, 1987, p.145-158

N.Palazzolo, Trasmissione di proveddimenti imperiali, dans IURA, 28, 1977, p.40-94

V.Parvan, Die Nationalität der Faufleute im rom.Kaiserreiche, Breslau, 1909

G.Pavis d'Escurac, Origo e résidence dans le monde du commerce sous le Haut-Empire, dans Ktèma, 13, 1988, p.57-68

H.G.Pflaum, Essai sur le cursus publicus sous le Haut-empire romain, Memoires présenté à

l'académie des Inscriptions et Belles lettres, tome XIV, 1940, 189-390

N. Purcell, dans O.Murray, La cité grecque d'Homère à Alexandre, OOO

E.S.Ramage, Urban problems in ancient Rome, dans R.T.Marchese, Aspects of Greek and Roman Urbanism : Essays on the Classical City, Oxford, 1983,

p. 61-92

R.Rebuffat, Une zone militaire et sa vie économique : le limes de Tripolitaine, dans Armée et fiscalité dans le monde antique, Paris, 1977, p.395-419

T.Reekmans et E.Van't Dack, A Bodleian Archive on corn transport, dans CE, 27, 1952, 149195

T.Reinach, Un code fiscal de l'Égypte romaine : le Gnomon de l'Idiologue, dans RHD, 43 (1919), 583-636 [Texte] ; 44 (1920),5-134 [commentaires]

O.W.Reinmuth, The Prefect of Egypt from Augustus to Diocletian, Leipzig 1935 (Klio, Beiheft XXXIV, n.folge, heft 21).

M.Reinhold, Usurpation of status and status symbols in the Roman Empire, dans Historia 1971, p.275-302

C.Ricci, Africani a Roma. Testimonianze epigrafiche di età imperiale di personnagi provenienti dal Nordafrica, dans Ant.Afr, 30, 1994, p.189-207

S.Riccobono, Il Gnomon, Palerme, 1950

G.Rickman, The Grain Trade under the Roman Empire, dans J.D’Arms et E.C.Kopff éd, 1980, p.261-275

L.Robert, Épitaphes métriques de médecins grecs, dans Hellenika II, 1946, p.102-108

L.Ross Taylor, Foreign groups in Roman politics of the late Republic, dans Hommages à J.Bidez et

F.Cumont, Latomus, vol.2, Bruxelles (s.d), p. 323-30

P.Salway, The Frontier People of Roman Britain, Cambridge U.Press, 1965

I.Savalli, I neocittadini nelle città ellenistiche. Note surlla concessione e acquisižione della politeia, dans

Historia 34, 1985, 400

A.C.Scafuro et Boegehold, Athenian Identity and Civic Ideology, Baltimore-Londres, 1994

V. Scarano Ussani, Le forme del privilegio, Beneficia e privilegia da Cesare e gli Antonini, Loffredo ed. Naples, 1992

B.Scardigli, I trattati romano-cartaginesi, Pise, SNS, 1991 
W.Seston et M.Euzennat, Un dossier de la chancellerie romaine : la Tabula Banasitana. Etude de diplomatique, dans CRAI, 1971, p.468-490 (= Scripta varia, Rome, 1980)

R.K.Sherk, Roman Documents from the Greek. East. Senatus consulta and Epistulae to the Age of Augustus, Baltimore, 1969

R.K.Sherk, Rome and the Greek East, Cambridge, 1984

S.M.Sherwin White, Ancient Archives : the Edict of Alexander to Priene. A Reappraisal, dans JHS 105, 1985, 69-89

S.E.Sidebotham, Roman Economic Policy in the Erythra Thalassa, 30 B.C.-A.D.217, Brill, 1986

S.E.Sidebotham et R.E.Zitterkopf, Stations and Towers in the Queseir-Nile Road, dans JEA 75, 1989, 155-89

P.J.Sijpesteijn, Small Notes on Bilingual Papyri, dans ZPE, 42, 1981, p.107-110 (ici 108-109)

W.G.Sinningen, The Officium of the Urban Prefecture during the Late Roman Empire, Rome, 1957

M.Sordi, L'espulsione degli Ebrei da Roma nel 49 d.C., dans Coercizione e mobilità umana nel mondo antico (Contributo dell'Istituto di st.antica, 21) Milan, 1995, p. 259-77

T.Spagnuolo-Vigorita, Lex portus Asiae : un nuovo documento sull' appalto delle imposte, dans I rapporti contrattuali con la pubblica amministrazione, Torino, 17-19 ott.1994, Naples, 1997, p.113-190

J.Suolahti, Legatio libera, dans Arctos, 6, 1970, p.113-119

W.Suder, Census populi : Bibliographie de la démographie de l'antiquité romaine, Bonn, 1988

P.R.Swarney, The Ptolemaic and Roman Idiologos, Toronto, 1970

R.Taubenschlag, The Law of Graeco-Roman Egypt in the light of the Papyri (332 B.C.-640 A.D.) ( Varsovie, 1948), Milan, 1972

J.Teixidor, Le tarif de Palmyre.I. Un commentaire de la version palmyréenne. Aula Orientalis I,2, Barcelone 1983, p.235-252

E.A. Thompson, Romans and Barbarians, 1982 ; cf dans Florilegium 2, 1980, p.71-88

S.L.Wallace, Taxation in Egypt from August to Diocletian, Princeton, 1938

C.R.Whittaker, Les frontières de l'empire romain, Paris-Besançon, 1989

W.Williams, Antoninus Pius and the Control of Provincial Embassies, dans Historia, 16, 1967, 47082

A.J.N.Wilson, Emigration from Italy in the Republican Age of Rome, New York, 1966 
